\title{
Convergence of Chorin-Marsden Product Formula in the Half-Plane
}

\author{
G. Benfatto * and M. Pulvirenti * \\ Dipartimento di Matematica, Università degli Studi “La Sapienza”, Piazzale A. Moro, 2, I-00185 \\ Roma, Italy
}

\begin{abstract}
Consider a viscous incompressible fluid in the half-plane and let $u_{t}$ be a solution of the Navier-Stokes equation. In this paper we prove that the product formula $\left(E_{t / n} G_{t / n} \phi u\right)^{n} u_{0}$, where $E_{t}$ is the Euler flow, $G_{t}$ is the heat flow and $\phi$ is a suitable operator describing the vorticity production due to the boundary, converges uniformly to $u_{t}$ in the limit $n \rightarrow \infty$.
\end{abstract}

\section{Introduction}

The time evolution of a slightly viscous incompressible fluid in the presence of obstacles exhibits features which are difficult to investigate both from an analytical and a numerical point of view, even in the simplest two-dimensional case. In particular, large gradients of the velocity field, localized near the boundary, make difficult the use of the conventional algorithms, which are essentially based on projections on low frequency quantities.

To overcome this difficulty, Chorin [1] developed an algorithm which can be briefly described, as suggested by Marsden [5], in the following way. Denoting by $E_{t}$ and $G_{t}$ the Euler and the heat semiflows, respectively $\left(G_{t}\right.$ satisfying suitable boundary conditions), then an approximation at time $t$ of the Navier-Stokes semiflow will be:

$$
\left(E_{t / n} G_{t / n} \phi\right)^{n},
$$

where $\phi$ is a suitable operator describing the vorticity production due to the boundary and making the nonslip boundary conditions (in general destroyed by $E_{t}$ and $G_{t}$ ) approximately satisfied.

The interest of the above method lies on the possibility of describing both $E_{t}$ and $G_{t}$ by means of particle dynamics (the particles are localized in points where the vorticity is sharply concentrated) thus taking into account, just from the very beginning, the high frequencies of the problem.

* Research supported by "Ministero della Pubblica Istruzione," CNR contract No. 84.00016.02 and GNFM 
Beyond the approximation problems of the single step algorithm, for which we address the reader to ref. [2] and references quoted therein, the convergence of the formula (1.1) to the Navier-Stokes semiflow, when $n \rightarrow \infty$, is a problem of conceptual and practical interest, See refs. [3-5] for a better introduction to the problem and for partial results concerning the convergence.

In the present paper we prove the convergence of the formula (1.1) in the simple situation of a fluid in the half-plane. Our proof is rather direct and explicit, thus we take a considerable advantage of the geometrical simplicity.

We remark that the techniques of this paper could be extended also to the halfspace case, obviously for short times only.

The estimates occurring in the proof are locally elementary. However, the general strategy may appear rather involved. We make use of the next section to outline the general ideas and to separate the single steps of the proof. The rest of the paper is devoted to the technical estimates necessary to prove the statements contained in Sect. 2.

\section{Results and Outline of the Proof}

Consider the Navier-Stokes initial value problem in the half-plane in terms of the vorticity:

$$
\left.\begin{array}{rl}
\partial_{t} \omega_{t}(x, y)+u_{t} \cdot \nabla \omega_{t}(x, y) & =\Delta \omega_{t}(x, y), \quad x \in \mathbb{R}, \quad y \geqq 0, \quad t \geqq 0, \\
\omega_{t} & =\operatorname{curl} u_{t}=-\partial_{y} u^{(1)}+\partial_{x} u^{(2)}, \\
u_{t}\left(x, 0^{+}\right) & =0
\end{array}\right\}
$$

$u_{t} \in \mathbb{R}^{2}$ is the velocity field, the density and the viscosity coefficients are assumed to be one and, finally, the initial value $u_{0}$ is a given function. If $u_{0}$ decays at infinity, the velocity field can be recovered by the vorticity $\omega_{t}$ by

$$
u_{t}=\nabla^{\perp} \Delta_{D}^{-1} \omega_{t}
$$

where $\nabla^{\perp}=\left(-\partial_{y}, \partial_{x}\right)$ and $\Delta_{D}$ denotes the Laplacian with Dirichlet boundary conditions.

The initial value problem (2.1) has been widely investigated and global existence and uniqueness theorems are known under suitable hypotheses on $u_{0}$ $[8,9]$.

The Chorin algorithm is defined, for $\varepsilon>0$, as

$$
\omega_{n}^{\varepsilon}=E_{\varepsilon} G_{\varepsilon} \phi \omega_{n-1}^{\varepsilon},
$$

where

$$
E_{\varepsilon} \omega=\omega-\varepsilon\left(\nabla^{\perp} \Delta_{D}^{-1} \omega\right) \cdot \nabla \omega
$$

is the infinitesimal Euler flow,

$$
G_{\varepsilon} \omega=e^{\varepsilon \Delta_{N}} \omega
$$

where $\Delta_{N}$ is the Laplacian with Neumann boundary conditions and, finally,

$$
\phi \omega_{n}^{\varepsilon}(x, y)=\omega_{n}^{\varepsilon}(x, y)+\varepsilon \tilde{f}_{n}^{\varepsilon}(x) \delta(y),
$$


where

$$
\varepsilon \tilde{f}_{n}^{\varepsilon}(x)=2 \partial_{y} \Delta_{D}^{-1} \omega_{n}^{\varepsilon}\left(x, 0^{+}\right) .
$$

The purpose of this paper is to prove that, under suitable hypotheses on $u_{0}$ (or $\omega_{0}$ ), the approximate velocity field converges to the solution of the Navier-Stokes equation, i.e.:

$$
\lim _{\substack{n \rightarrow \infty \\ \varepsilon \rightarrow \infty \\ n \varepsilon=t}} \nabla^{\perp} \Delta_{D}^{-1} \omega_{n}^{\varepsilon}(x, y)=u_{t}(x, y)
$$

for each $t$, uniformly in $x$ and $y$, where $u_{t}$ solves uniquely the initial value problem (2.1) with initial datum $u_{0}$.

We spend some words to explain the meaning of the algorithm (2.3). It is rather obvious that the limit

$$
\lim _{n \rightarrow \infty}\left(E_{t / n} G_{t / n}\right)^{n} \omega_{0}
$$

if it exists, does not solve the problem (2.1) but only the analogous one with boundary condition

$$
\partial_{y} \omega_{t}\left(x, 0^{+}\right)=0
$$

$\left[u_{t}^{(2)}(x, 0)=0\right.$ is automatically satisfied by $(2.2)$, while $u_{t}^{(1)}(x, 0) \neq 0$ in general]. The insertion of the vortex sheet given by (2.7) has the goal of restoring the correct boundary conditions at each time step. This action is actually equivalent to extend the velocity field in the lower half-plane in an odd and even way for $u^{(1)}$ and $u^{(2)}$, respectively ( $\omega$ is also extended as an even function consequently), therefore $\varepsilon \tilde{f}^{\varepsilon}$ expresses the jump discontinuity of $u^{(1)}$. The subsequent application of $G_{\varepsilon}$ will smear out such discontinuity and the nonslip boundary conditions will be approximately satisfied.

The vorticity produced by the action of $\phi$ at each time step is expected to be of the order of $\varepsilon$ and this explains the somehow arbitrary $\varepsilon$ appearing in (2.6). Therefore, $\tilde{f}^{\varepsilon}$ can be interpreted as the rate of vorticity production of the process.

We give a flavour of the difficulty in proving the limit (2.8). It is natural, as a preliminary step, to look for bounds on $\omega_{n}^{\varepsilon}$ which are uniform in $\varepsilon$. If one tries to do it iteratively, a naive estimate of the amount of vorticity produced at each time step gives [see Eq. (4.18) below] two terms. One is linear in the previous total vorticity, but is of order $\sqrt{\varepsilon}$; the other one is of order $\varepsilon$, but is bilinear. Therefore, to avoid a catastrophic error production, we look at formula (2.3) globally in time, by means of an appropriate expansion which is the time discretization of the usual perturbation of the Stokes semiflow. Namely, after some trivial algebra, it is easy to realize that the following identity holds:

$$
\omega_{n}^{\varepsilon}=S_{n}^{\varepsilon} \omega_{0}-\sum_{k=0}^{n-1} \varepsilon S_{n-k-1}^{\varepsilon} b_{k}^{\varepsilon} \cdot \nabla S_{1}^{\varepsilon} \omega_{k}^{\varepsilon},
$$

where

$$
S_{n}^{\varepsilon}=\left(G_{\varepsilon} \phi\right)^{n}
$$

and

$$
b_{k}^{\varepsilon}=\nabla^{\perp} \Delta_{D}^{-1} S_{1}^{\varepsilon} \omega_{k}^{\varepsilon} .
$$


Moreover we have, for an arbitrary initial vorticity profile $\gamma$ :

$$
S_{n}^{\varepsilon} \gamma=G_{n \varepsilon} \gamma+\sum_{k=1}^{n-1} \varepsilon G_{\varepsilon(n-k)}\left(f_{k}^{\gamma, \varepsilon} \delta\right)+G_{n \varepsilon}\left(a^{\gamma} \delta\right),
$$

where

$$
a^{\gamma}(x)=2 \partial_{y} \Delta_{D}^{-1} \gamma\left(x, 0^{+}\right)
$$

is the initial sheet of vorticity to insert if $\gamma$ does not satisfy the boundary condition at time zero [like $b_{k}^{\varepsilon} \cdot \nabla S_{1}^{\varepsilon} \omega_{k}^{\varepsilon}$ in formula $\left.(2.11)\right], f_{k}^{\gamma, \varepsilon} \delta(x, y)$ and $a^{\gamma} \delta(x, y)$ stand for $f_{k}^{\gamma, \varepsilon}(x) \delta(y)$ and $a^{\gamma}(x) \delta(y)$, respectively, and, finally, the function $f_{k}^{\gamma, \varepsilon}$ is determined by the condition

$$
\varepsilon f_{k}^{\gamma, \varepsilon}=2 \partial_{y} \Delta_{D}^{-1}\left[G_{k \varepsilon} \omega_{0}+\sum_{s=1}^{k-1} \varepsilon G_{\varepsilon(k-s)}\left(f_{s}^{\gamma, \varepsilon} \delta\right)+G_{k \varepsilon}\left(a^{\gamma} \delta\right)\right]\left(\cdot, 0^{+}\right) .
$$

The formal limits of (2.11), (2.14), and (2.15) for $\varepsilon \rightarrow 0, n \rightarrow \infty, n \varepsilon=t$ are

$$
\omega_{t}=S_{t} \omega_{0}+\int_{0}^{t} d s S_{t-s}\left(u_{s} \cdot \nabla\right) \omega_{s}
$$

where

$$
S_{t} \gamma=G_{t} \gamma+\int_{0}^{t} d s G_{t-s}\left(f_{s}^{\gamma} \delta\right)+G_{t}\left(a^{\gamma} \delta\right)
$$

and

$$
\partial_{y} \Delta_{D}^{-1}\left(G_{t} \gamma+\int_{0}^{t} d s G_{t-s} f_{s}^{\gamma} \delta+G_{t} a^{\gamma} \delta\right)\left(\cdot, 0^{+}\right)=0 .
$$

Since we are interested in the convergence of $\omega_{n}^{\varepsilon}$ given by (2.11), it is natural to investigate first the structure of the formal limit expressed above by Eqs. (2.17)-(2.19). This analysis has been performed by the authors of the present paper in ref. [6]. In particular, Eq. (2.19) can be explicitly solved (due to the simple geometry of the problem). The results concerning Eq. (2.19) are reviewed as Step 1. As Step 2 we try to carry out the same analysis for the identity (2.16). Unfortunately in this case we do not have an explicit expression for $f_{k}^{\gamma, \varepsilon}$ but only bounds following suitable algebraic manipulations. On the basis of such estimates we are now able to give uniform bounds on $S_{n}^{\varepsilon} \omega_{0}$ and $\omega_{n}^{\varepsilon}$ (the Stability Theorem). This is Step 3. At this point we notice that the stability for $S_{n}^{\varepsilon} \omega_{0}$ (but also for $\omega_{n}^{\varepsilon}$ ) can be obtained in the energy norm, rather easily, realizing that the operator $\phi$ is energy preserving and the whole algorithm dissipates energy (see ref. [3]). Nevertheless, in such norm, while the convergence of the linear problem is quite natural, the approach to the non-linear problem seems more problematic. In fact the non-linear limit problem (2.17)-(2.19) can be more efficiently investigated (see ref. [6]) by the use of different and, in some sense, more natural norms, which will be used also in this paper.

As Steps 4 and 5 we prove the convergence of $S_{t}^{\varepsilon}$ and $\omega_{n}^{\varepsilon}$, making use of the above preparation.

We now analyze the various steps in more detail. 
Step 1. Equation (2.19) may be better understood in terms of the Fourier transform. Denoting by $\hat{F}$ the Fourier transform of any function $F: \mathbb{R} \rightarrow \mathbb{R}$ or $F: \mathbb{R} \times \mathbb{R}^{+} \rightarrow \mathbb{R}$ with respect to the $x$ variable, we have:

$$
\begin{gathered}
\left(\partial_{x} \Delta_{D}^{-1} F\right)(p, y)=-i \varepsilon(p) / 2 \int_{0}^{\infty} d y_{1}\left(e^{-|p|\left|y-y_{1}\right|}-e^{-|p|\left|y+y_{1}\right|}\right) \hat{F}\left(p, y_{1}\right) \\
-\left(\partial_{y} \Delta_{D}^{-1} F\right)(p, y)=1 / 2 \int_{0}^{\infty} d y_{1}\left(e^{-|p|\left|y-y_{1}\right|} \varepsilon\left(y_{1}-y\right)+e^{-|p|\left|y+y_{1}\right|} \varepsilon\left(y_{1}+y\right)\right) \hat{F}\left(p, y_{1}\right),
\end{gathered}
$$

where $\varepsilon(x), x \in \mathbb{R}$, is the sign function.

By (2.19) we have (omitting the index $\gamma$ on $f$ and $a$ for simplicity):

$$
\begin{aligned}
\hat{m}_{t}(p) & =2 \int_{0}^{t} d s \int_{0}^{\infty} d y\left[e^{-|p| y-p^{2}(t-s)-y^{2} /(t-s)} / \sqrt{4 \pi(t-s)}\right] \hat{f}_{s}(p) \\
& =\int_{0}^{t} d s \varphi\left(\sqrt{p^{2}(t-s)}\right) \hat{f}_{s}(p),
\end{aligned}
$$

where, by definition,

$$
\begin{gathered}
\varphi(x)=(2 / \sqrt{\pi}) \int_{x}^{\infty} d z e^{-z^{2}} \\
\hat{m}_{t}(p)=-2 \int_{0}^{\infty} d y e^{-|p| y} e^{-p^{2 t}} \int_{-\infty}^{+\infty} d y_{1} g_{t}\left(y-y_{1}\right) \hat{\gamma}(p, y)-2 \int_{0}^{\infty} d y e^{-|p| y} e^{-p^{2} t} g_{t}(y) \hat{a}(p),
\end{gathered}
$$

and

$$
g_{t}(y)=e^{-y^{2} / 4 t} / \sqrt{4 \pi t} .
$$

In formula (2.24) (we shall do the same very often in the rest of the paper) we have denoted by the same symbol the even (with respect to the $y$ variable) extension of $\hat{\gamma}$ in the whole plane in such a way that $G_{t}$ can be easily expressed in terms of the free heat kernel.

Differentiating Eq. (2.22) with respect to the $t$ variable, we have:

$$
\hat{h}_{t}=[(1-T) \hat{f}]_{t},
$$

where

$$
\hat{h}_{t}=\partial_{t} \hat{m}_{t}
$$

and

$$
(\hat{T f})_{t}(p)=\int_{0}^{t} d s \partial_{s} \varphi\left(\sqrt{p^{2}(t-s)}\right) \hat{f}_{s}(p)=(|p| / \sqrt{\pi}) \int_{0}^{t} d s / \sqrt{t-s}\left(e^{-p^{2}(t-s)} \hat{s}_{s}(p)\right) .
$$

The Neumann series associated to Eq. (2.26) can be explicitly calculated and the result is

$$
\hat{f}_{t}(p)=\left(p^{2}+\partial_{t}\right) \hat{m}_{t}(p)+(|p| / \sqrt{\pi}) \int_{0}^{t} d s / \sqrt{t-s}\left(e^{-p^{2}(t-s)}\left(p^{2}+\partial_{s}\right) \hat{m}_{s}(p)\right) .
$$


Formula (2.29) has been obtained in [6], where the Stokes problem was investigated in this light. In Sect. 3 we give (also for the sake of completeness) an alternative, and in some sense more natural, proof of Eq. (2.29).

Step 2. One can hope to deal with the approximated problem (2.16) in the same way. In fact, by the same algebra, we obtain:

$$
\hat{m}_{n \varepsilon}(p)=\sum_{m=1}^{n} \varepsilon \varphi\left(\sqrt{\varepsilon p^{2}(n-m)}\right) \hat{f}_{m}^{\varepsilon}(p)
$$

from which

$$
\hat{f}_{n}^{\varepsilon}=\widehat{h}_{n}^{\varepsilon}+\sum_{k=1}^{n-1}\left(T_{\varepsilon}^{k} \hat{h}^{\varepsilon}\right)_{n},
$$

where

$$
\hat{h}_{n}^{\varepsilon}=(D \hat{m})_{k \varepsilon}=\left(\hat{m}_{k \varepsilon}-\hat{m}_{(k-1) \varepsilon}\right) / \varepsilon
$$

and

$$
\left(\hat{T_{\varepsilon} h}\right)_{n}(p)=\sum_{m=1}^{n-1} \delta_{n-1-m}(\lambda) \hat{h}_{m}(p),
$$

where $\lambda=\varepsilon p^{2}$ and

$$
\delta_{k}(\lambda)=2 / \sqrt{\pi} \int_{\sqrt{k \lambda}}^{\sqrt{(k+1) \lambda}} d z e^{-z^{2}} .
$$

To estimate $f$ it is convenient to separate the first two terms of the right-hand side of (2.31) from the rest, denoted by $R_{n}(p)$. We have

$$
\begin{aligned}
R_{n}(p) & =\sum_{k=2}^{n-1}\left(T_{\varepsilon}^{k} \hat{h^{\varepsilon}}\right)_{n} \\
& =\sum_{k=2}^{n-1} \sum_{m_{k}=1}^{n-k} \sum_{m_{k-1}=m_{k}+1}^{n-(k-1)} \ldots \sum_{m_{1}=m_{2}+1}^{n-1} \hat{h}_{m_{k}}^{\varepsilon} \delta_{n-1-m_{1}} \delta_{m_{1}-1-m_{2}} \ldots \delta_{m_{k-1}-1-m_{k}} \\
& =\sum_{m=1}^{n-2} \hat{h}_{m}^{k} \sum_{k=2}^{n-m} \sum_{\substack{s_{1} \ldots s_{k} \geqq 1 \\
\Sigma_{i} s_{i}=n-m}} \delta_{s_{1}-1} \ldots \delta_{s_{k}-1}=\sum_{m=1}^{n-2} \hat{h}_{m}^{\varepsilon} J_{n-m}(\lambda)
\end{aligned}
$$

where

$$
J_{m}(\lambda)=\sum_{k=2}^{m} \sum_{\substack{s_{1}+\ldots+s_{k}=m-k \\ s_{i} \geq 0}} \delta_{s_{1}}(\lambda) \ldots \delta_{s_{k}}(\lambda) .
$$

The basic estimate is the following

\section{Proposition 1.}

$$
J_{m}(\lambda) \leqq c \lambda .
$$

Proof in Sect. 3.

By virtue of Proposition 1 we can prove, more or less straightforwardly: 
Proposition 2.

$$
\left|\hat{f}_{n}^{\varepsilon}\right| \leqq c(|p|+1 / \sqrt{\varepsilon n}) \sup _{y}|\hat{\gamma}(p, y)|
$$

Proof in Sect. 3.

Remark. The bound (2.38) is not so good as in the limit situation for which we have, after a direct inspection of (2.29) (see Sect. 5 or [6])

$$
\left|\hat{f}_{t}\right|(p) \leqq(c / \sqrt{t}) \sup _{y}|\hat{\gamma}(p, y)| .
$$

A similar bound for $\hat{f}_{t}^{\varepsilon}$ (if true) is very difficult to obtain: the estimate (2.39) is a consequence of the lucky circumstance that Eq. (2.26) is exactly solvable.

Step 3. We find convenient (see also ref. [6]) the use of the following norms:

$$
\begin{array}{rlrl}
\|F\| & =\int d p \sup _{y} \mid \hat{F}(p, y), & F: \mathbb{R}^{2} \rightarrow \mathbb{R}^{m}, \quad m=1,2, \\
\|f\|=\int d p|\hat{f}(p)|, & f: \mathbb{R}^{1} \rightarrow \mathbb{R}^{1} .
\end{array}
$$

To obtain uniform bounds on $\left\|\omega_{n}^{\varepsilon}\right\|$ and $\left\|b_{n}^{\varepsilon}\right\|$, we first need to estimate $\left\|S_{n}^{\varepsilon} \omega_{0}\right\|$ [see (2.11)]. This estimate is rather easy by virtue of Proposition 2. More involved is the estimate of $\left\|S_{n}^{\varepsilon} \gamma\right\|$, where $\gamma=\left(b_{k}^{\varepsilon} \cdot \nabla\right) S_{\varepsilon}^{1} \omega_{k}^{\varepsilon}$. In fact the same strategy in this case leads to bounds involving norms of derivatives of $S_{\varepsilon}^{1} \omega_{k}^{\varepsilon}$. Nevertheless, using the fact that $b_{k}^{\varepsilon}$ is almost zero for $y=0$ and the fundamental theorem of calculus, one can estimate $\left\|S_{n}^{\varepsilon} \gamma\right\|$ by a multilinear form in $\omega_{k}^{\varepsilon}$ so arriving to an integral inequality of the type $Y_{n}^{\varepsilon} \leqq Y_{0}+c \sum_{k=1}^{n-1}\left(Y_{k}^{\varepsilon}\right)^{\alpha}$, for some $\alpha>1$, where $Y_{n}^{\varepsilon}$ is a positive quantity larger than $\left\|b_{n}^{\varepsilon}\right\|$ and $\left\|\omega_{n}^{\varepsilon}\right\|$. From this we obtain a stability result, for short times. More precisely we have:

Lemma 1. Let $b=\nabla^{\perp} \Delta_{D}^{-1} S_{1}^{\varepsilon} \gamma$. Then

$$
\left|\hat{b}^{(1)}(p, 0)\right| \leqq c \sqrt{\varepsilon} \sup _{y}|\hat{\gamma}(p, y)|
$$

Moreover,

$$
\left|\hat{b}^{(i)}(p, y)\right| \leqq c\left(\sqrt{\varepsilon} \sup _{y}|\hat{\gamma}(p, y)|+y \sup _{y}\left|S_{1}^{\varepsilon \hat{\gamma}}(p, y)\right|\right) .
$$

Proof in Sect. 4.

The following proposition is the basic ingredient for the stability and convergence. In proving such a proposition we improve the estimate (2.38) making use of Lemma 1.

Proposition 3. The following bound holds:

$$
\left\|S_{n}^{\varepsilon} \gamma\right\| \leqq c(\|\gamma\|+\|a\| / \sqrt{n \varepsilon}) .
$$

Furthermore, assuming $\gamma$ of the form $\gamma=(b \cdot \nabla) F$, where $b$ is a vector field satisfying $\nabla \cdot b=0$, we have:

$$
\begin{gathered}
\left\|S_{n}^{\varepsilon} \gamma\right\| \leqq c\left(\left\|\partial_{x}(b F)\right\|+\|b F\| / \sqrt{n \varepsilon}\right) \\
\left\|\nabla^{\perp} \Delta_{D}^{-1} S_{n}^{\varepsilon} \gamma\right\| \leqq c\|b F\|
\end{gathered}
$$


Moreover, if

$$
|\hat{b}(p, y)| \leqq c(\sqrt{\varepsilon}+y) H(p)
$$

for some function $H \geqq 0$, then the following is true:

$$
\left\|S_{n}^{\varepsilon} \gamma\right\| \leqq c\left(\int d p H(p)\|F\|+1 / \sqrt{\varepsilon n}\|b F\|\right) .
$$

Proof in Sect. 4.

The above proposition, combined with Lemma 1, allows us to obtain the following stability theorem.

Theorem 1. Let $u_{0}$ be the initial velocity field satisfying the boundary conditions $u_{0}\left(x, 0^{+}\right)=0$. Let $\omega_{0}=\operatorname{curl} u_{0}$ and

$$
M_{k}^{\varepsilon}=\sup _{m \leqq k} \max \left\{\left\|\omega_{m}^{\varepsilon}\right\|,\left\|S_{1}^{\varepsilon} \omega_{m}^{\varepsilon}\right\|,\left\|b_{m}^{\varepsilon}\right\|\right\} .
$$

Then there exist $t_{0}, \varepsilon_{0}$, and $c$ such that

$$
M_{k}^{\varepsilon} \leqq c\left(t_{0}\right)\left(\left\|\omega_{0}\right\|+\left\|u_{0}\right\|\right)
$$

for $k \varepsilon \leqq t_{0}$ and $\varepsilon<\varepsilon_{0}$.

Proof in Sect. 4.

Step 4. To prove the convergence of the Stokes algorithm we need to introduce norms involving derivatives. In fact, we need to compare $\hat{f}$ with $\hat{f}^{\varepsilon}$. Since $\varepsilon$ appears always in the combination $\varepsilon p^{2}$, to prove that the difference $\hat{f}-\hat{f}_{\varepsilon}$ is infinitesimal in $\varepsilon$ we have to control some extra $|p|^{\alpha}$ appearing in the estimate. Putting, for any scalar or vector valued function $F$ defined on $\mathbb{R}^{2}$ :

$$
\|F\|^{(n, m)}=\max _{\substack{0 \leqq i \leqq n \\ 0 \leqq j \leqq m}}\left\|\partial_{x}^{i} \partial_{y}^{j} F\right\|,
$$

and, for any real valued function defined on $\mathbb{R}^{1}$

$$
\|F\|^{(n)}=\max _{0 \leqq i \leqq n}\left\|\partial_{x}^{i} F\right\|,
$$

we have:

Theorem 2. For sufficiently small $\varepsilon>0$ and $t=n \varepsilon, n \geqq 1$,

$$
\begin{gathered}
\left\|\left(S_{t}-S_{n}^{\varepsilon}\right) \gamma\right\| \leqq c(1+t)\left(\varepsilon^{1 / 4}\left(\|\gamma\|^{(3,0)}+\|\gamma\|^{(2,1)}\right)+(\varepsilon / t)^{1 / 4}\|a\|^{(2)}\right), \\
\left\|\nabla^{\perp} \Delta_{D}^{-1}\left(S_{t}-S_{n}^{\varepsilon}\right) \gamma\right\|+\left\|\nabla^{\perp} \Delta_{D}^{-1}\left(S_{t}-S_{n}^{\varepsilon}\right) \gamma\right\| \\
\leqq \varepsilon^{1 / 4} c(1+t)\left(\|\gamma\|^{(2,0)}+\|\gamma\|^{(1,1)}+(\varepsilon / t)^{1 / 4}\|a\|^{(1)}\right) .
\end{gathered}
$$

Proof in Sect. 6.

The proof of Theorem 2 is based on the layer theory discussed in Step 1 and Step 2 and on some regularity properties of the Stokes semiflow to which Sect. 5 is devoted. 
Step 5. We are now in position to prove the convergence of $b_{n}^{\varepsilon}$ to $u_{n \varepsilon}$. This is based on the expansion $(t=n \varepsilon)$ :

$$
\begin{aligned}
\omega_{t}-S_{1}^{\varepsilon} \omega_{n}^{\varepsilon}= & \left(S_{n \varepsilon}-S_{n+1}^{\varepsilon}\right) \omega_{0}+\left[\int_{0}^{t} d s S_{t-s}\left(u_{s} \cdot \nabla\right) \omega_{s}-\sum_{k=0}^{n-1} \varepsilon S_{(n-k) \varepsilon}\left(u_{k \varepsilon} \cdot \nabla\right) \omega_{k \varepsilon}\right] \\
& +\sum_{k=0}^{n-1} \varepsilon\left(S_{(n-k) \varepsilon}-S_{n-k}^{\varepsilon}\right)\left(u_{k \varepsilon} \cdot \nabla\right) \omega_{k \varepsilon} \\
& +\sum_{k=0}^{n-1} \varepsilon S_{n-k}^{\varepsilon}\left[\left(u_{k \varepsilon}-b_{k}^{\varepsilon}\right) \cdot \nabla \omega_{k \varepsilon}\right] \\
& +\sum_{k=0}^{n-1} \varepsilon S_{n-k}^{\varepsilon}\left(b_{k}^{\varepsilon} \cdot \nabla\right)\left(\omega_{k \varepsilon}-S_{1}^{\varepsilon} \omega_{k}^{\varepsilon}\right),
\end{aligned}
$$

and a similar expansion for the velocity field.

Since we know, by [6], regularity properties of $\omega_{t}$, we can apply Theorem 2 to the first and third terms of the right-hand side of (2.54).

By Theorem 1 we have uniform bounds on $\left\|b_{k}^{\varepsilon}\right\|$. Therefore, we establish a linear integral inequality for $\max \left\{\left\|\omega_{t}-S_{1}^{\varepsilon} \omega_{n}^{\varepsilon}\right\|,\left\|u_{t}-b_{n}^{\varepsilon}\right\|\right\}$ to prove the convergence for short times. Arbitrary times are recovered by standard arguments since we know that $\left\|\omega_{t}\right\|$ and $\left\|u_{t}\right\|$ are bounded in any finite time interval. The precise statement is the following:

Theorem 3. Suppose $\left\|\omega_{0}\right\|^{(6,2)}<+\infty, u_{0} \in W_{2}^{2}, u_{0}\left(x, 0^{+}\right)=0$. Then, for all $t>0$, $0<\alpha<1 / 4$

$$
\lim _{\substack{n \rightarrow \infty \\ \varepsilon \rightarrow 0 \\ n \varepsilon=t}}\left\|\omega_{n}^{\varepsilon}-\omega_{t}\right\| \varepsilon^{-\alpha}=\lim _{\substack{n \rightarrow \infty \\ \varepsilon \rightarrow 0 \\ n \varepsilon=t}}\left\|b_{n}^{\varepsilon}-u_{t}\right\| \varepsilon^{-\alpha}=0 .
$$

Proof in Sect. 7.

Some additional remarks are needed.

The approximate rate of production of vorticity of the full nonlinear problem, i.e. $\tilde{f}_{n}^{\varepsilon}$ in Eq. (2.6), has not been explicitly estimated. A direct estimate of $\tilde{f}_{n}^{\varepsilon}$, which seems difficult in our norms, has been avoided by perturbing the approximated Stokes semiflow $S_{n}^{\varepsilon}$. Nevertheless, in the limiting situation, the explicit knowledge of the solution of Eq. (2.26) allows a direct estimate of the rate of production of vorticity (see estimate (2.45) of ref. [6]) which is interesting in itself.

More general geometry seems difficult to deal with. The basic reason is that the operator $T$ appearing in (2.26) has $\|\cdot\|$-norm larger than one: this, obviously, creates problems for general situations.

The estimates in this paper are certainly not optimal. The rate of convergence is $\varepsilon^{\alpha}$ with $\alpha=1 / 4$. This can be improved up to $\alpha=1 / 2$ by the use of the present techniques with some extra technical effort. Further improvements, if possible, could require new ideas.

A similar product formula was studied in ref [7], the main difference being that in (1.1) $G_{t / n} \phi$ is replaced by the operator $S_{t / n}$, the Stokes semiflow (this greatly simplifies the proof of convergence). The results obtained in ref. [7] are weaker as regards the regularity properties, but are valid for any smooth bounded domain. Our techniques could be applied (in the case of the half-plane or the half-space) as well to this product formula. 
We conclude this section by fixing some conventions. We shall denote by $c$ any numerical positive constant and by $c(\xi, \eta, \ldots)$ any positive constant depending only on the quantities $\xi, \eta, \ldots$. All the constants appearing in this paper are computable, but we shall not give their values.

\section{The Stokes Problem: Technical Details}

Let us consider the Neumann series associated to Eq. (2.26):

$$
\hat{f}_{t}(p)=\hat{h}_{t}(p)+\sum_{k=1}^{\infty}\left(T^{\hat{k}} h\right)_{t}(p), \quad \hat{h}_{t}=\partial_{t} \hat{m}_{t}
$$

where $T^{k}$ is the $k^{\text {th }}$ power of the operator $T$ defined by Eq. $(2.28)$ and $\hat{m}_{t}(p)$ is defined by Eq. (2.24). We want to show that this series can be explicitly calculated [under suitable hypotheses on the function $\left.\hat{m}_{t}(p)\right]$.

Notice that, if $k \geqq 2$ :

$$
\left(T^{\hat{k}} h\right)(p)=\int_{0}^{t} d s \hat{h}_{s}(p) e^{-p^{2}(t-s)} I_{k}(t-s),
$$

where

$$
\begin{gathered}
I_{k}(t-s)=(|p| / \sqrt{\pi})^{k} \int_{t \geqq s_{1} \geqq s_{2} \geqq \ldots \geqq s} d s_{1} \ldots d s_{k-1}\left(t-s_{1}\right)^{-1 / 2}, \\
\left(s_{1}-s_{2}\right)^{-1 / 2} \ldots\left(s_{k-1}-s\right)^{-1 / 2}=(t-s)^{k / 2-1}|p|^{k} / \Gamma(k / 2) \\
\Gamma(u)=\int_{0}^{\infty} d x x^{u-1} e^{-x}
\end{gathered}
$$

Equations (3.3), (3.4), and the formula

$$
n !=\left(2^{n} / \sqrt{\pi}\right) \Gamma(n / 2+1) \Gamma(n / 2+1 / 2)
$$

imply that

Therefore,

$$
\begin{aligned}
\sum_{k=2}^{\infty} I_{k}(t-s) & =p^{2} \sum_{n=0}^{\infty} \sqrt{p^{2}(t-s)^{n}} / \Gamma(n / 2+1) \\
& =p^{2} \sum_{n=0}^{\infty}\left(\sqrt{p^{2}(t-s)^{n}} / n !\right)\left(2^{n} / \sqrt{\pi}\right) \int_{0}^{\infty} d x x^{\frac{n}{2}-\frac{1}{2}} e^{-x} \\
& \left.=p^{2} \sum_{n=0}^{\infty}\left(2 \sqrt{p^{2}(t-s)}\right)^{n} / n !\right)\left(2^{2} / \sqrt{\pi}\right) \int_{0}^{\infty} d z z^{n} e^{-z^{2}} \\
& =p^{2}(2 / \sqrt{\pi}) \int_{0}^{\infty} d z e^{-z^{2}+2 z \sqrt{p^{2}(t-s)}} .
\end{aligned}
$$

$$
\begin{aligned}
\sum_{k=2}^{\infty}\left(T^{\hat{k}} h\right)_{t}(p) & =p^{2} \int_{0}^{t} d s \hat{h}_{s}(p)(2 / \sqrt{\pi}) \int d z e^{-\left(z-\sqrt{\left.p^{2}(t-s)\right)^{2}}\right.} \\
& =p^{2} \int_{0}^{t} d s \hat{h}_{s}(p)\left[2-\varphi\left(\sqrt{p^{2}(t-s)}\right)\right]
\end{aligned}
$$

where $\varphi(x)$ is the error function [see Eq. (2.23)]. 
Equations (3.1) and (3.7) imply that

$$
\begin{aligned}
\hat{f}_{t}(p)= & \partial_{t} \hat{m}_{t}+(|p| / \sqrt{\pi}) \int_{0}^{t} d s / \sqrt{t-s}\left(e^{-p^{2}(t-s)} \partial_{s} \hat{m}_{s}(p)\right) \\
& +p^{2} \int_{0}^{t} d s \partial_{s} \hat{m}_{s}(p)\left[2-\varphi\left(\sqrt{p^{2}(t-s)}\right)\right]
\end{aligned}
$$

Equation (2.29) follows from Eq. (3.8) and an integration by parts in the last term of its right-hand side.

It is worth noticing that the use of Eq. (2.29) in place of Eq. (3.8) was essential in the treatment of the Navier-Stokes equation explained in ref. [6]. However, in the discrete case, there is a formula similar to Eq. (3.8) (in the sense that it obeys similar bounds) for $f$, but it seems difficult to obtain a formula similar to Eq. (2.29). This forced us to use, in the discrete case, a different approach, with respect to the continuum one, in order to get good estimates for $\omega_{n}^{\varepsilon}$ and $b_{n}^{\varepsilon}$ (see also the comment following Proposition 2).

The analogue of Eq. (3.8) in the discrete case is [see Eqs. (2.31) and (2.35)]:

$$
\hat{f}_{n}^{\varepsilon}(p)=\hat{h}_{n}^{\varepsilon}(p)+\left(T_{\varepsilon} \hat{h}\right)_{n}(p)+R_{n}^{\varepsilon}(p),
$$

and the claim that Eq. (3.9) allows essentially the same bounds, uniformly in $\varepsilon$, as Eq. (3.8) is an immediate consequence of Proposition 1, that we are going to prove.

Proof of Proposition 1. If $m=2$, by Eq. (2.34):

$$
J_{2}(\lambda)=\delta_{0}(\lambda)^{2} \leqq(4 / \pi) \lambda
$$

If $m \geqq 3$, we can write:

$$
J_{m}(\lambda)=S_{m}(\lambda)-S_{m-1}(\lambda)
$$

where, for $m \geqq 2$ :

$$
S_{m}(\lambda)=\sum_{k=2}^{m} J_{k}(\lambda)
$$

If we put $\hat{h}_{m}^{\varepsilon}=1$ in Eq. $(2.35)$, we obtain

where

$$
S_{n-1}(\lambda)=\sum_{m=1}^{n-2} J_{n-m}(\lambda)=\sum_{k=2}^{n-1} I_{n, k}^{\varepsilon}
$$

$$
I_{n, k}^{\varepsilon}=\sum_{m_{1}=k}^{n-1} \delta_{n-1-m_{1}}(\lambda) \sum_{m_{2}=k-1}^{m_{1}-1} \delta_{m_{1}-1-m_{2}}(\lambda) \ldots \sum_{m_{k}=1}^{m_{k}-1-1} \delta_{m_{k-1}-1-m_{k}}(\lambda) .
$$

Let us observe now that $\delta_{k}(\lambda)$ can be written in the following way:

Therefore,

$$
\delta_{k}(\lambda)=(1 / \sqrt{\pi}) \int_{\lambda k}^{\lambda(k+1)}(d u / \sqrt{u}) e^{-u} .
$$

$$
\begin{aligned}
I_{n, k}^{\varepsilon}= & (1 / \sqrt{\pi})^{k} \sum_{m_{1}=k}^{n-1} \int_{\lambda m_{1}}^{\lambda\left(m_{1}+1\right)} d s_{1}\left(\lambda n-s_{1}\right)^{-1 / 2} e^{-\left(\lambda n-s_{1}\right)} \ldots \\
& \cdots \sum_{m_{k}=1}^{m_{k}-1} \int_{\lambda m_{k}}^{\lambda\left(m_{k}+1\right)} d s_{k}\left(\lambda m_{k-1}-s_{k}\right)^{-1 / 2} e^{-\left(\lambda m_{k}-1-s_{k}\right)} .
\end{aligned}
$$


An important point in the proof is that the sums in Eq. (3.16) can be calculated "almost" exactly. Let us consider the simplest case $k=2$.

$$
\begin{aligned}
I_{n, 2}^{\varepsilon}= & (1 / \pi) \sum_{m_{1}=2}^{n-1} \int_{\lambda m_{1}}^{\lambda\left(m_{1}+1\right)} d s_{1}\left(\lambda n-s_{1}\right)^{-1 / 2} e^{-\left(\lambda n-s_{1}\right)} \\
& \times \int_{\lambda}^{\lambda m_{1}} d s_{2}\left(\lambda m_{1}-s_{2}\right)^{-1 / 2} e^{-\left(\lambda m_{1}-s_{2}\right)} \\
= & (1 / \pi) \sum_{m_{1}=2}^{n-1} \int_{\lambda m_{1}}^{\lambda\left(m_{1}+1\right)} d s_{1}\left(\lambda n-s_{1}\right)^{-1 / 2} e^{-\left(\lambda n-s_{1}\right)} \\
& \times \int_{\lambda+\varepsilon^{(1)}}^{s_{1}} d s_{2}\left(s_{1}-s_{2}\right)^{-1 / 2} e^{-\left(s_{1}-s_{2}\right)} \\
= & (1 / \pi) \int_{2 \lambda}^{n \lambda} d s_{1}\left(\lambda n-s_{1}\right)^{-1 / 2} \int_{\lambda+\varepsilon^{(1)}}^{s_{1}} d s_{2}\left(s_{1}-s_{2}\right)^{-1 / 2} e^{-\left(\lambda n-s_{2}\right)},
\end{aligned}
$$

where $\varepsilon^{(1)}=s_{1}-\left[s_{1} / \lambda\right]$ is a non-negative function of $s_{1}$ smaller than $\lambda$.

In a similar way it is easy to show that

$$
\begin{aligned}
I_{n, k}^{\varepsilon}= & (1 / \sqrt{\pi})^{k} \int_{k \lambda}^{n \lambda} d s_{1}\left(\lambda n-s_{1}\right)^{-1 / 2} \int_{(k-1) \lambda+\varepsilon^{(1)}}^{s_{1}} d s_{2}\left(s_{1}-s_{2}\right)^{-1 / 2} \ldots \\
& \cdots \int_{\lambda+\varepsilon^{(k-1)}}^{s_{k}-1} d s_{k}\left(s_{k-1}-s_{k}\right)^{-1 / 2} e^{-\left(\lambda n-s_{k}\right)},
\end{aligned}
$$

where $\varepsilon^{(j)}, j=1, \ldots, k-1$, is a function of $s_{1}, s_{2}, \ldots, s_{j}$, such that

$$
0 \leqq \varepsilon^{(j)} \leqq j \lambda .
$$

Equations (3.3), (3.18), and (3.19) imply that

$$
\int_{k \lambda}^{n \lambda} d s e^{-(\lambda n-s)}(\lambda n-s)^{\frac{k}{2}-1} / \Gamma(k / 2) \leqq I_{n, k}^{\varepsilon} \leqq \int_{0}^{\lambda n} d s e^{-(\lambda n-s)}(\lambda n-s)^{\frac{k}{2}-1} / \Gamma(k / 2) .
$$

It is worth noticing, at this point, that, for the purposes of this paper, it should be sufficient to prove an upper bound for $\left|\left(T_{\varepsilon}^{k} \hat{h}^{\varepsilon}\right)_{n}\right|$ similar to the one appearing in Eq. (3.20), that is:

$$
\mid\left(T_{\varepsilon}^{k} \hat{h}_{n}(p)\left|\leqq \int_{0}^{\lambda n} d s\right| \hat{h}_{s}(p) \mid e^{-(\lambda n-s)}(\lambda n-s)^{\frac{k}{2}-1} / \Gamma(k / 2) .\right.
$$

In fact, this should allow to bound $R_{n}^{\varepsilon}(p)$ by the corresponding continuum expression (3.7) [with $\left|\hat{h}_{s}(p)\right|$ in place of $\hat{h}_{s}(p)$ ]. However, this is possible only if $\left|\hat{h}_{s}(p)\right|$ is an increasing function of $s$, which is not the case in the applications that we are interested on. Nevertheless, the more refined result of Proposition 1, in which we use also the lower bound of (3.20), is sufficient for our needs.

Let us now complete the proof of Proposition 1. By the same calculations leading to Eq. (3.7) and by Eq. (3.13):

Moreover,

$$
S_{n-1}(\lambda) \leqq \int_{0}^{\lambda n} d s(2-\varphi(\sqrt{s}))
$$

$$
\begin{aligned}
S_{n-1}(\lambda) & \geqq \sum_{k=2}^{n-1}\left[\int_{0}^{\lambda n} d s e^{-(\lambda n-s)}(\lambda n-s)^{\frac{k}{2}-1} / \Gamma(k / 2)-\int_{0}^{\lambda k} d s(\lambda n-s)^{\frac{k}{2}-1} / \Gamma(k / 2)\right] \\
& \geqq \int_{0}^{\lambda n} d s(2-\varphi(\sqrt{s}))-\Delta_{n}^{1}-\Delta_{n}^{2}
\end{aligned}
$$


where

$$
\begin{aligned}
\Delta_{n}^{1}= & \sum_{k=n}^{\infty} \int_{0}^{\lambda n} d s e^{-(\lambda n-s)}(\lambda n-s)^{\frac{k}{2}-1} / \Gamma(k / 2) \\
= & \int_{0}^{\lambda n} d s e^{-(\lambda n-s)}(\lambda n-s)^{\frac{n}{2}-1} / \Gamma(n / 2-1) \\
& \times \sum_{k=0}^{\infty}\left[(\lambda n-s)^{k / 2} / \Gamma(k / 2+1)\right] \beta(k / 2+1, n / 2-1), \\
\Delta_{n}^{2}= & \sum_{k=2}^{n-1} \int_{0}^{\lambda k} d s(\lambda n-s)^{\frac{k}{2}-1} / \Gamma(k / 2)=\sum_{k=2}^{n-1}(\lambda k)^{k / 2} / \Gamma(k / 2+1) .
\end{aligned}
$$

We defined, as usual:

$$
\beta(u, v)=\Gamma(u) \Gamma(v) / \Gamma(u+v) .
$$

Since $\beta(u, v)$ is decreasing in $u$ and $v$, if $n \geqq 3$, we have

$$
\begin{aligned}
\Delta_{n}^{1} & \leqq \beta(1,1 / 2) \int_{0}^{\lambda n} d s\left[(\lambda n-s)^{\frac{n}{2}-1} / \Gamma(n / 2-1)\right](2-\varphi(\sqrt{\lambda n-s})) \\
& \leqq 2 \beta(1,1 / 2)(\lambda n)^{n / 2} / \Gamma(n / 2) .
\end{aligned}
$$

It is easy to prove that there exists $\lambda_{0} \leqq 1$ and a positive constant $c$ such that, for $n \geqq 3$ :

$$
\begin{aligned}
& \Delta_{n}^{1} \leqq c_{0} \lambda \eta_{n}, \quad \eta_{n} \underset{n \rightarrow \infty}{\longrightarrow} 0, \\
& \Delta_{n}^{2} \leqq c_{0} \lambda .
\end{aligned}
$$

Therefore, if $\lambda \leqq \lambda_{0}$ and $m \geqq 3$, by Eqs. (3.11), (3.22), (3.23), and (3.28), there exists $c_{1}>0$ such that

$$
J_{m}(\lambda) \leqq \int_{0}^{\lambda(m+1)} d s(2-\varphi(\sqrt{s}))-\int_{0}^{\lambda m} d s(2-\varphi(\sqrt{s}))+\Delta_{m}^{1}+\Delta_{m}^{2} \leqq c_{1} \lambda .
$$

The case $\lambda \geqq \lambda_{0}$ can be treated by induction. Notice that, by Eq. (2.36):

$$
J_{m}(\lambda)=\sum_{s=0}^{m-2} \delta_{s} \delta_{m-2-s}+\sum_{s=0}^{m-3} \delta_{s} J_{m-1-s} .
$$

Let us suppose that there exists a constant $c$ such that, if $2 \leqq k \leqq m-1$ and $\lambda \geqq \lambda_{0}$ :

$$
J_{k} \leqq c \lambda \sum_{s=2}^{k} e^{-(s-2) \lambda} .
$$

If we use the bound (3.31) in the right-hand side of Eq. (3.30), we find, using Eq. (3.15), if $m \geqq 3$ :

$$
\begin{aligned}
J_{m}(\lambda) \leqq & \delta_{0} \delta_{m-2}+\sum_{s=1}^{m-2}(1 / \pi) \int_{\lambda(s-1)}^{\lambda s} d t_{1}\left(\lambda(m-2)-t_{1}\right)^{-1 / 2} e^{-\left[\lambda(m-2)-t_{1}\right]} \\
& \times \int_{\lambda s}^{\lambda(s+1)} d t_{2} t_{2}^{-1 / 2} e^{-t_{2}}+c \lambda \sum_{s=2}^{m-1} e^{-(s-2) \lambda} \sum_{k=0}^{\infty} \delta_{k} .
\end{aligned}
$$


Since $\sum_{k=0}^{\infty} \delta_{k}=1$, we can write:

$$
\begin{aligned}
J_{m}(\lambda) \leqq & (4 / \pi) \lambda e^{-(m-2) \lambda} /(\sqrt{m-1}+\sqrt{m-2} \\
& +(\lambda / \pi) e^{-\lambda(m-2)} \sum_{s=1}^{m-2} d t_{1}\left(\lambda(m-2)-t_{1}\right)^{-1 / 2} t_{1}^{-1 / 2} \\
& +c \lambda \sum_{s=2}^{m-1} e^{-(s-2) \lambda} \\
\leqq & (1+4 / \pi) \lambda e^{-\lambda(m-2)}+c \lambda \sum_{s=2}^{m-1} e^{-(s-2) \lambda}
\end{aligned}
$$

Therefore, the bound (3.31) is true also for $k=m$, if $c \geqq 1+4 / \pi$. By (3.10), for any $m \geqq 2$ and any $\lambda \geqq \lambda_{0}$, we have:

$$
J_{m}(\lambda) \leqq c \lambda \sum_{s=0}^{\infty} e^{-\lambda_{0} s}=c \lambda /\left(1-e^{-\lambda_{0}}\right) .
$$

Proof of Proposition 2. Equations (2.31), (2.33), (2.35), and (2.37) imply that

$$
\left|\hat{f}_{n}^{\varepsilon}\right| \leqq\left|\hat{h}_{n}^{\varepsilon}\right|+\sum_{m=1}^{n-1} \delta_{n-1-m}\left|\hat{h}_{m}^{\varepsilon}\right|+c \sum_{m=1}^{n-2} \lambda\left|\hat{h}_{m}^{\varepsilon}\right|
$$

Moreover, by Eqs. (2.24) and (2.32):

$$
\left|\hat{h}_{k}^{\varepsilon}\right|=\left|\left(\hat{m}_{k \varepsilon}-\hat{m}_{(k-1) \varepsilon}\right) / \varepsilon\right| \leqq(1 / \varepsilon) \int_{(k-1) \varepsilon}^{k \varepsilon} d \sigma\left|\partial_{\sigma} \hat{m}_{\sigma}\right|
$$

and

$$
\partial_{\sigma} \hat{m}_{\sigma}=2|p| e^{-p^{2} \sigma} \int_{-\infty}^{+\infty} d y_{1} g_{\sigma}\left(y_{1}\right) \hat{\gamma}\left(p, y_{1}\right)-2|p| e^{-p^{2} \sigma} g_{\sigma}(0) \int_{-\infty}^{+\infty} d y_{1} e^{-|p|\left|y_{1}\right|} \hat{\gamma}\left(p, y_{1}\right),
$$

where we did some integrations by parts and used the even symmetry of $\hat{\gamma}$ as a function of $y$. Equation (3.37) implies that

$$
\left|\partial_{\sigma} \hat{m}_{\sigma}\right| \leqq c(|p|+1 / \sqrt{\sigma}) e^{-p^{2} \sigma} \sup _{y}|\hat{\gamma}(p, y)| .
$$

Then, by Eq. (3.36), if $n \geqq 1$ :

$$
\left|h_{n}^{\varepsilon}\right| \leqq c(|p|+1 / \sqrt{\varepsilon n}) e^{-\varepsilon p^{2}(n-1)} \sup _{y}|\hat{\gamma}(p, y)| .
$$

Equations (3.35) and (3.39) imply that

$$
\left|\hat{f}_{n}^{\varepsilon}\right| \leqq\left|\hat{h}_{n}^{\varepsilon}\right|+c \lambda\left|\hat{h}_{n}^{\varepsilon}\right|+c \sup _{y}|\hat{\gamma}(p, y)| K_{n},
$$

where

$$
K_{n}=\sum_{m=1}^{n-1} \delta_{n-1-m}(\lambda)(|p|+1 / \sqrt{\varepsilon m}) e^{-\lambda(m-1)}+\sum_{m=2}^{n-2} \lambda(|p|+1 / \sqrt{\varepsilon m}) e^{-\lambda(m-1)} .
$$


By Eqs. (3.36) and (3.38),

$$
\begin{aligned}
\lambda\left|h_{1}^{\varepsilon}\right| & \leqq c p^{2} \int_{0}^{\varepsilon} d \sigma(|p|+1 / \sqrt{\sigma}) e^{-p^{2} \sigma} \sup _{y}|\hat{\gamma}(p, y)| \\
& \leqq c \sup _{y}|\hat{\gamma}(p, y)||p| \int_{0}^{p^{2} \varepsilon} d x(1+1 / \sqrt{x}) e^{-x} \\
& \leqq c|p| \sup _{y}|\hat{\gamma}(p, y)| .
\end{aligned}
$$

Moreover, by Eqs. (3.15) and (3.41),

$$
\begin{aligned}
K_{n} \leqq & |p|\left(1+\sum_{m=2}^{n-2} \lambda e^{-\lambda(m-1)}\right) \\
& +\sum_{m=1}^{n-1}(2 / \sqrt{\pi}) e^{-\lambda(n-2)} \sqrt{\lambda} /[(\sqrt{n-m}+\sqrt{n-m-1}) \sqrt{\varepsilon m}] \\
& +\sum_{m=2}^{n-2} \lambda e^{-\lambda(m-1)} / \sqrt{\varepsilon m} \\
\leqq & |p|\left(1+\lambda e^{-\lambda} /\left(1-e^{-\lambda}\right)\right)+(2 / \sqrt{\pi}) e^{-\lambda(n-2)}|p| \sum_{m=1}^{n-1} m^{-1 / 2} /(\sqrt{n-m}+\sqrt{n-m-1}) \\
& +|p| \sum_{m=2}^{n-2} \int_{(m-1) \lambda}^{m \lambda} d u / \sqrt{u} e^{-(u-\lambda)} .
\end{aligned}
$$

Proposition 2 follows from Eqs. (3.39), (3.40), (3.42), (3.43), and the bounds

$$
\begin{gathered}
\sum_{m=1}^{n-1} m^{-1 / 2} /(\sqrt{n-m}+\sqrt{n-m-1}) \leqq \sum_{m=1}^{n-1} \int_{m-1}^{m} d u /(\sqrt{u} \sqrt{n-1-u}) \\
=\int_{0}^{1} d x /(\sqrt{x} \sqrt{1-x})=\pi, \\
\sum_{m=2}^{n-2} \int_{(m-1) \lambda}^{m \lambda}(d u / \sqrt{u}) e^{-(u-\lambda)} \leqq \int_{0}^{\infty}(d u / \sqrt{u+\lambda}) e^{-u} \leqq \int_{0}^{\infty}(d u / \sqrt{u}) e^{-u} .
\end{gathered}
$$

In the following section, in order to prove Proposition 3, we shall need a more refined version of Proposition 2, valid if $\gamma$ is of the form

$$
\gamma=(b \cdot \nabla) F,
$$

where $b$ is a divergenceless vector field.

Proposition 4. If $\gamma$ is of the form (3.46), then

$$
\left|f_{n}^{\varepsilon}\right| \leqq c\left(|p|+1 / \sqrt{\varepsilon n)} \sup _{y}|p \cdot b \hat{F}(p, y)| .\right.
$$

Moreover, if

$$
|\hat{b}(p, y)| \leqq c(\sqrt{\varepsilon}+y) H(p),
$$

then

$$
\left|\hat{f}_{n}^{\varepsilon}\right| \leqq c(|p|+1 / \sqrt{\varepsilon n}) \int d q H(p-q) \sup _{y}|\hat{F}(q, y)|
$$


Proof. Equations (3.37), (3.46), and $\nabla \cdot b=0$ imply that

$$
\left|\partial_{\sigma} \hat{m}_{\sigma}\right| \leqq c(|p|+1 / \sqrt{\sigma}) e^{-p^{2} \sigma} \sup _{y}|p b \hat{F}(p, y)|,
$$

which leads to Eq. (3.47), proceeding as before. If $b$ satisfies the bound (3.48), we can write:

$$
\left|\partial_{\sigma} \hat{m}_{\sigma}\right| \leqq c[|p|(|p|+1 / \sqrt{\sigma})(\sqrt{\varepsilon}+\sqrt{\sigma})+1 / \sqrt{\sigma}] e^{-p^{2} \sigma} \bar{H}(p)
$$

where

$$
\bar{H}(p)=\int d q H(p-q) \sup _{y}|\hat{F}(q, y)| .
$$

Equation (3.51) implies that

$$
\left|\partial_{\sigma} \hat{m}_{\sigma}\right| \leqq c \bar{H}(p)\left[|p|(1+\sqrt{\varepsilon / \sigma}) e^{-p^{2} \sigma / 2}+1 / \sqrt{\sigma} e^{-p^{2} \sigma}\right],
$$

which gives very easily, for $n \geqq 1$, using Eq. (3.36):

$$
\left|\widehat{h}_{n}^{\varepsilon}\right| \leqq c \bar{H}(p)\left[|p| e^{-p^{2} \varepsilon(n-1) / 2}+1 / \sqrt{\varepsilon n} e^{-p^{2} \varepsilon(n-1)}\right] .
$$

Equations (3.35) and (3.54) imply that

$$
\left|\hat{f}_{n}^{\varepsilon}\right| \leqq\left|\bar{h}_{n}^{\varepsilon}\right|+c \lambda\left|\widehat{h}_{1}^{\varepsilon}\right|+c \bar{H}(p) \bar{K}_{n},
$$

where $\bar{K}_{n}$ is very similar to $K_{n}$ [see Eq. (3.41)] and allows essentially the same bounds. The only important difference is in the term $\lambda\left|\hat{h}_{1}^{\varepsilon}\right|$, which can now be estimated in the following way:

$$
\begin{aligned}
\lambda\left|\hat{h}_{1}^{\varepsilon}\right| & \leqq c p^{2} \bar{H}(p) \int_{0}^{\varepsilon} d \sigma\left[|p|(1+\sqrt{\varepsilon / \sigma}) e^{-p^{2} \sigma / 2}+{ }^{1} / \sqrt{\sigma} e^{-p^{2} \sigma}\right] \\
& \leqq c \bar{H}(p)|p|(\sqrt{\lambda}+1) .
\end{aligned}
$$

Equation (3.56) allows us to get the bound (3.49) only if $\lambda \leqq \lambda_{0}$, where $\lambda_{0}$ is an arbitrary constant greater than 0 . If $\lambda \geqq \lambda_{0}$, it is necessary to use another strategy. Observe that the $\sqrt{\lambda}$ in Eq. (3.56) comes only from the first term in the right-hand side of Eq. (3.54) and that it is possible to write $\hat{h}_{n}^{\varepsilon}$ in the form $\hat{h}_{n}^{\varepsilon}=\hat{h}_{1, n}^{\varepsilon}+\hat{h}_{2, n}^{\varepsilon}$, so that

$$
\begin{aligned}
& \left|\hat{h}_{1, n}^{\varepsilon}\right| \leqq c \bar{H}(p)|p| e^{-\lambda(n-1) / 2}, \\
& \left|\hat{h}_{2, n}^{\varepsilon}\right| \leqq c \bar{H}(p) / \sqrt{\varepsilon n} e^{-\lambda(n-1)} .
\end{aligned}
$$

This decomposition of $h$ implies a corresponding decomposition $\hat{f}_{n}^{\varepsilon}=\hat{f}_{1, n}^{\varepsilon}+\hat{f}_{2, n}^{\varepsilon}$. $\hat{f}_{2, n}^{\varepsilon}$ can be bounded as in the proof of Proposition 2 for any $\lambda$. As regards $\hat{f}_{1, n}^{\varepsilon}$, we observe that, by Eq. (2.30),

$$
\hat{f}_{1, n}^{\varepsilon}=\hat{h}_{1, n}^{\varepsilon}+\sum_{m=1}^{n-1} \delta_{n-m-1} \hat{f}_{1, m}^{\varepsilon}, \quad n \geqq 1 .
$$

Let us suppose that, if $1 \leqq m \leqq n-1$,

$$
\left|\hat{f}_{1, m}^{\varepsilon}\right| \leqq c \bar{H}(p)|p| \sum_{k=1}^{m} e^{-\lambda(k-1) / 2} .
$$


Then, by Eqs. (3.59) and (3.57),

$$
\begin{aligned}
\left|\hat{f}_{1, n}^{\varepsilon}\right| & \leqq c \bar{H}(p)|p| e^{-\lambda(n-1) / 2}+c \bar{H}(p)|p| \sum_{k=1}^{n-1} e^{-\lambda(k-1) / 2} \sum_{m=1}^{\infty} \delta_{m} \\
& =c \bar{H}(p)|p| \sum_{k=1}^{n} e^{-\lambda(k-1) / 2} .
\end{aligned}
$$

Therefore, Eq. (3.60) is true also for $m=n$ and it implies, if $\lambda \geqq \lambda_{0}>0$,

$$
\left|\hat{f}_{1, n}^{\varepsilon}\right| \leqq c|p| \bar{H}(p)\left(1-e^{-\lambda_{0} / 2}\right)^{-1} .
$$

This completes the proof of Proposition 4.

\section{The Stability Bound}

Proof of Proposition 3. Equation (2.14) and Proposition 2 imply that

$$
\begin{aligned}
\left\|S_{n}^{\varepsilon} \gamma\right\| \leqq & \left\|G_{n \varepsilon} \gamma\right\|+c \sum_{k=1}^{n-1} \varepsilon \int d p e^{-\varepsilon p^{2}(n-k)} / \sqrt{\varepsilon(n-k)}(|p|+1 / \sqrt{\varepsilon k}) \sup _{y}|\hat{\gamma}(p, y)| \\
& +\int d p(1 / \sqrt{4 \pi n \varepsilon}) e^{-n \varepsilon p^{2}}\left|\hat{a}^{\gamma}(p)\right| \\
\leqq & \|\gamma\|+\left\|a^{\gamma}\right\| / \sqrt{4 \pi n \varepsilon}+c\|\gamma\|\left\{\sup _{\lambda \geqq 0} \sum_{k=1}^{n-1} \lambda e^{-\lambda k} / \sqrt{\lambda k}+\sum_{k=1}^{n-1} 1 / \sqrt{k(n-k)}\right\} .
\end{aligned}
$$

The bound (2.43) follows from Eqs. (4.1), (3.44), and the inequality

$$
\sum_{k=1}^{n-1} \lambda e^{-\lambda k} / \sqrt{\lambda k} \leqq \sum_{k=1}^{\infty} \int_{(k-1) \lambda}^{k \lambda}(d u / \sqrt{u}) e^{-u}=\int_{0}^{\infty}(d u / \sqrt{u}) e^{-u}
$$

Let us now suppose that $\gamma=(b \cdot \nabla) F$. By Eqs. (2.14) and (3.47) we have (by some integrations by parts and using $\nabla \cdot b=0$ ), if $n \geqq 1$ :

$$
\left\|S_{n}^{\varepsilon} \gamma\right\| \leqq c / \sqrt{n \varepsilon}\|b F\|+\left\|a^{\gamma}\right\| / \sqrt{4 \pi n \varepsilon}+c\left\|\partial_{x}(b F)\right\| .
$$

Furthermore,

$$
\hat{a}^{\gamma}(p)=-2 \int_{0}^{\infty} d y e^{-|p| y} \widehat{\nabla(b F)}(p, y),
$$

which implies

$$
\left\|a^{\gamma}\right\| \leqq c\|b F\| \text {. }
$$

The bound (2.44) follows from Eqs. (4.3) and (4.5).

In a similar way we obtain Eq. (2.47), using the bound (3.49).

Finally, by using Eqs. (2.14), (2.20), (2.21), and (3.47), we have:

$$
\begin{aligned}
\left\|\nabla^{\perp} \Delta_{D}^{-1} S_{n}^{\varepsilon} \gamma\right\| \leqq & c\left\|a^{\gamma}\right\|+c\|b F\|+c \sum_{k=1}^{n-1} \varepsilon \int d p e^{-\varepsilon p^{2}(n-k)}\left(p^{2}+|p| / \sqrt{\varepsilon k}\right) \\
& \times \sup _{y}|\hat{b F}(p, y)| \leqq c\|b F\| .
\end{aligned}
$$


Proof of Lemma 1. By Eq. (2.14),

$$
\hat{S_{1}^{\varepsilon}} \gamma=\hat{G_{\varepsilon}} \gamma-2 g_{\varepsilon}(y) e^{-p^{2} \varepsilon} \int_{0}^{\infty} d y e^{-|p| y_{1}} \hat{\gamma}(p, y) .
$$

Then, if $b=\nabla^{\perp} \Delta_{D}^{-1} S_{1}^{\varepsilon} \gamma$

$$
\hat{b}^{(1)}(p, 0)=\int_{0}^{\infty} d y e^{-|p| y}\left(\hat{S_{1}^{\varepsilon}} \gamma\right)(p, y) \underset{\varepsilon \rightarrow 0}{\longrightarrow} 0,
$$

which becomes, using Eq. (4.7):

$$
\hat{b^{(1)}}(p, 0)=\int_{0}^{\varepsilon} d \sigma \int_{0}^{\infty} d y e^{-|p| y} \partial_{\sigma}\left\{\hat{G_{\sigma}} \gamma(p, y)-2 g_{\sigma}(y) e^{-p^{2} \sigma} \int_{0}^{\infty} d y_{1} e^{-|p| y_{1}} \hat{\gamma}\left(p, y_{1}\right)\right\},
$$

which implies very easily the bound (2.41).

Equation (2.42) follows from Eqs. (4.9), (2.20), (2.21), the trivial identity

$$
\hat{b}^{(i)}(p, y)=\hat{b}^{(i)}(p, 0)+\int_{0}^{y} d y_{1} \hat{b}^{(i)}\left(p, y_{1}\right)
$$

and the fact that $\hat{b}^{(2)}(p, 0)=0$.

Proof of Theorem 1. First we shall estimate $\omega_{n}^{\varepsilon}$. By Eq. (2.11), if $n \geqq 1$,

$$
\left\|\omega_{n}^{\varepsilon}\right\| \leqq\left\|S_{n}^{\varepsilon} \omega_{0}\right\|+\left\|\varepsilon b_{n-1}^{\varepsilon} \nabla S_{1}^{\varepsilon} \omega_{n-1}^{\varepsilon}\right\|+\sum_{k=0}^{n-2} \varepsilon\left\|S_{n-k-1}^{\varepsilon} b_{k}^{\varepsilon} \cdot \nabla S_{1}^{\varepsilon} \omega_{k}^{\varepsilon}\right\| .
$$

If $\omega_{0}$ satisfies the boundary conditions, we can apply the bound (2.43) with $a^{\gamma}=0$ to obtain

$$
\left\|S_{n}^{\varepsilon} \omega_{0}\right\| \leqq c\left\|\omega_{0}\right\|
$$

Furthermore, Eq. (2.13), Lemma 1, and Proposition 3 imply that

$$
\begin{aligned}
& \sum_{k=0}^{n-2} \varepsilon\left\|S_{n-k-1}^{\varepsilon} b_{k}^{\varepsilon} \nabla S_{1}^{\varepsilon} \omega_{k}^{\varepsilon}\right\| \\
& \quad \leqq c \sum_{k=0}^{n-2} \varepsilon\left[\left\|S_{1}^{\varepsilon} \omega_{k}^{\varepsilon}\right\|\left(\left\|\omega_{k}^{\varepsilon}\right\|+\left\|S_{1}^{\varepsilon} \omega_{k}^{\varepsilon}\right\|\right)+\left\|b_{k \varepsilon}\right\| \cdot\left\|S_{1}^{\varepsilon} \omega_{k}^{\varepsilon}\right\| / \sqrt{\varepsilon(n-k-1)}\right] \\
& \quad \leqq c \sum_{k=0}^{n-2} \varepsilon\left(M_{k}^{\varepsilon}\right)^{2}(1+1 / \sqrt{\varepsilon(n-k-1)})
\end{aligned}
$$

where $M_{k}^{\varepsilon}$ is defined as in Eq. (2.48).

In order to bound $\left\|\omega_{n}^{\varepsilon}\right\|$, we have still to bound $\left\|\varepsilon b_{n-1}^{\varepsilon} \cdot \nabla S_{1}^{\varepsilon} \omega_{n-1}^{\varepsilon}\right\|$. If $n=1$, we have:

$$
\left\|\varepsilon b_{0}^{\varepsilon} \cdot \nabla S_{1}^{\varepsilon} \omega_{0}\right\|=\left\|\varepsilon b_{0}^{\varepsilon} \cdot \nabla G_{\varepsilon} \omega_{0}\right\| \leqq c \sqrt{\varepsilon}\left\|b_{0}^{\varepsilon}\right\|\left\|\omega_{0}\right\| \leqq c \sqrt{\varepsilon}\left(M_{0}^{\varepsilon}\right)^{2} .
$$

If $n>1$, we observe that

$$
S_{1}^{\hat{\varepsilon}} \omega_{n-1}^{\varepsilon}=G_{\varepsilon}^{\hat{\varepsilon}} \omega_{n-1}^{\varepsilon}-2 e^{-\varepsilon p^{2}} g_{\varepsilon}(y) \tilde{b_{n-1}^{\varepsilon(1)}}(p, 0),
$$

where

$$
\widetilde{b_{n-1}^{\varepsilon(1)}}=-\partial_{y} \Delta_{D}^{-1} \omega_{n-1}^{\varepsilon} .
$$


Furthermore, Eq. (2.11) implies that

$$
\omega_{n-1}^{\varepsilon}=S_{1}^{\varepsilon} \omega_{n-2}^{\varepsilon}-\varepsilon b_{n-2}^{\varepsilon} \cdot \nabla S_{1}^{\varepsilon} \omega_{n-2}^{\varepsilon} .
$$

By Eqs. (4.16) and (4.17) we have, using Eqs. (2.13), (2.21), and (2.41)

$$
\begin{aligned}
\left|\widetilde{b_{n-1}^{\varepsilon(1)}}(p, 0)\right| & \leqq\left|b_{n-2}^{\varepsilon(1)}(p, 0)\right|+\varepsilon\left|\int_{0}^{\infty} d y e^{-|p| y}\left(\nabla \widehat{b_{n-2}^{\varepsilon} S_{1}^{\varepsilon} \omega_{n-2}^{\varepsilon}}\right)(p, y)\right| \\
& \leqq c \sqrt{\varepsilon} \sup \left|\omega_{n-2}(p, y)\right|+c \varepsilon \sup _{y}\left|\widehat{b_{n-2}^{\varepsilon} S_{1}^{\varepsilon} \omega_{n-2}^{\varepsilon}}(p, y)\right| .
\end{aligned}
$$

Equations (4.15) and (4.18) imply that

$$
\begin{aligned}
\left\|\varepsilon b_{n-1}^{\varepsilon} \cdot \nabla S_{1}^{\varepsilon} \omega_{n-1}^{\varepsilon}\right\| & \leqq c \sqrt{\varepsilon}\left\|b_{n-1}^{\varepsilon}\right\|\left[\left\|\omega_{n-1}^{\varepsilon}\right\|+\left\|\omega_{n-2}^{\varepsilon}\right\|+\sqrt{\varepsilon}\left\|b_{n-2}^{\varepsilon}\right\|\left\|S_{1}^{\varepsilon} \omega_{n-2}^{\varepsilon}\right\|\right] \\
& \leqq c \sqrt{\varepsilon}\left(M_{n-1}^{\varepsilon}\right)^{2}\left(1+\sqrt{\varepsilon} M_{n-1}^{\varepsilon}\right) .
\end{aligned}
$$

Putting together the bounds (4.12), (4.13), (4.14), and (4.19), we can write:

$$
\left\|\omega_{n}^{\varepsilon}\right\| \leqq c\left\{\left\|\omega_{0}\right\|+\sum_{k=0}^{n-1} \varepsilon\left(M_{k}^{\varepsilon}\right)^{2}\left(1+M_{k}^{\varepsilon}\right)+\sum_{k=0}^{n-1} \varepsilon\left(M_{k}^{\varepsilon}\right)^{2} / \sqrt{\varepsilon(n-k)}\right\} .
$$

To complete the proof of Theorem 1, we need to estimate also $S_{1}^{\varepsilon} \omega_{n}^{\varepsilon}$ and $b_{n}^{\varepsilon}$. By Eq. (2.11),

$$
S_{1}^{\varepsilon} \omega_{n}^{\varepsilon}=S_{n+1}^{\varepsilon} \omega_{0}-\sum_{k=0}^{n-1} \varepsilon S_{n-k}^{\varepsilon} b_{k}^{\varepsilon} \cdot \nabla S_{1}^{\varepsilon} \omega_{k}^{\varepsilon},
$$

which implies, using Eqs. (4.12) and Proposition 3:

$$
\begin{aligned}
\left\|S_{1}^{\varepsilon} \omega_{n}^{\varepsilon}\right\| & \leqq c\left\|\omega_{0}\right\|+c \sum_{k=0}^{n-1} \varepsilon\left[\left\|S_{1}^{\varepsilon} \omega_{k}^{\varepsilon}\right\|\left(\left\|\omega_{k}^{\varepsilon}\right\|+\left\|S_{1}^{\varepsilon} \omega_{k}^{\varepsilon}\right\|\right)+\left\|b_{k}^{\varepsilon}\right\|\left\|S_{1}^{\varepsilon} \omega_{k}\right\| / \sqrt{\varepsilon(n-k)}\right] \\
& \leqq c\left\|\omega_{0}\right\|+c \sum_{k=0}^{n-1} \varepsilon\left(M_{k}^{\varepsilon}\right)^{2}(1+1 / \sqrt{\varepsilon(n-k)})
\end{aligned}
$$

Equation (4.21) implies also, together with Eq. (2.13), that

$$
b_{n}^{\varepsilon}=\nabla^{\perp} \Delta_{D}^{-1} S_{n+1}^{\varepsilon} \omega_{0}-\sum_{k=0}^{n-1} \varepsilon \nabla^{\perp} \Delta_{D}^{-1} S_{n-k}^{\varepsilon} b_{k}^{\varepsilon} \cdot \nabla S_{1}^{\varepsilon} \omega_{k}^{\varepsilon} .
$$

By Eqs. (2.14), (2.20), and (2.21), we can write:

$$
\left\|\nabla^{\perp} \Delta_{D}^{-1} S_{n+1}^{\varepsilon} \omega_{0}\right\| \leqq\left\|\nabla^{\perp} \Delta_{D}^{-1} G_{(n+1) \varepsilon} \omega_{0}\right\|+\int d p \sum_{k=1}^{n-1} \varepsilon e^{-\varepsilon(n-k) p^{2}}\left|\hat{f}_{k}^{\omega_{0}, \varepsilon}(p)\right|
$$

Notice now that, since $u_{0}(x, 0)=0$,

$$
G_{t} \omega_{0}=G_{t} \varepsilon_{y} \nabla^{\perp} \cdot u_{0}=\nabla^{\perp} \cdot G_{t}\left(\varepsilon_{y} u_{0}\right)
$$

where $\varepsilon_{y}$ is the sign function in the $y$ variable. $G_{t}\left(\varepsilon_{y} u_{0}\right)$ is, for $y \geqq 0$, a divergenceless vector field; therefore, by using Eqs. (2.20) and (2.21) with $F=\nabla^{\perp} G_{t}\left(\varepsilon_{y} u_{0}\right)$ and integrating by parts, we obtain:

$$
\left\|\nabla^{\perp} \Delta_{D}^{-1} G \omega_{0}\right\| \leqq c\left\|G_{t}\left(\varepsilon_{y} u_{0}\right)\right\| \leqq c\left\|u_{0}\right\|
$$


Furthermore, by Eq. (2.38)

$$
\begin{aligned}
\int d p \sum_{k=1}^{n-1} \varepsilon e^{-\varepsilon(n-k) p^{2}}\left|\hat{f}_{k}^{\omega_{0}, \varepsilon}(p)\right| & \leqq c\left\|\omega_{0}\right\| \sum_{k=1}^{n-1} \varepsilon(1 / \sqrt{\varepsilon(n-k)}+1 / \sqrt{\varepsilon k}) \\
& \leqq c \sqrt{\varepsilon(n-1)}\left\|\omega_{0}\right\| .
\end{aligned}
$$

We have also, using Eqs. (2.14), (2.20), and (2.21):

$$
\begin{aligned}
& \left|\nabla^{\perp} \Delta_{D}^{-1} S_{n-k}^{\varepsilon}\left(b_{k}^{\varepsilon} \cdot \nabla S_{1}^{\varepsilon} \omega_{k}^{\varepsilon}\right)(p, y)\right| \leqq \sup _{y_{1}}\left|G_{\varepsilon(n-k)} b_{k}^{\varepsilon} S_{1}^{\varepsilon} \omega_{k}^{\varepsilon}\left(p, y_{1}\right)\right| \\
& \quad+\sum_{r=1}^{n-k-1} \varepsilon e^{-\varepsilon(n-k-r) p^{2}}\left|\hat{f}_{r}^{\gamma, \varepsilon}(p)\right|+\left|\hat{a}^{\gamma}(p)\right|
\end{aligned}
$$

where $\gamma=b_{k}^{\varepsilon} \nabla S_{1}^{\varepsilon} \omega_{k}^{\varepsilon}$. By Eqs. (2.42) and (3.49),

$$
\begin{aligned}
& \int d p \sum_{r=1}^{n-k-1} \varepsilon e^{-\varepsilon(n-k-r) p^{2}}\left|\hat{f}_{r}^{\gamma, \varepsilon}(p)\right| \\
& \quad \leqq c\left(\left\|\omega_{k}^{\varepsilon}\right\|+\left\|S_{1}^{\varepsilon} \omega_{k}^{\varepsilon}\right\|\right)\left\|S_{1}^{\varepsilon} \omega_{k}^{\varepsilon}\right\| \cdot \sum_{r=1}^{n-k-1} \varepsilon(1 / \sqrt{\varepsilon(n-k-r)}+1 / \sqrt{\varepsilon r}) \\
& \quad \leqq c \sqrt{\varepsilon(n-k-1)}\left(M_{k}^{\varepsilon}\right)^{2} .
\end{aligned}
$$

Finally,

$$
\int d p\left|a^{\gamma}(p)\right| \leqq c\left\|b_{k}^{\varepsilon}\right\|\left\|S_{1}^{\varepsilon} \omega_{k}^{\varepsilon}\right\| \leqq c\left(M_{k}^{\varepsilon}\right)^{2} .
$$

Equations (4.24), (4.26), (4.27), (4.29), and (4.30) imply that

$$
\left\|b_{n}^{\varepsilon}\right\| \leqq c\left\|u_{0}\right\|+c \sqrt{n \varepsilon}\left\|\omega_{0}\right\|+c \sum_{k=0}^{n-1} \varepsilon\left(M_{k}^{\varepsilon}\right)^{2}(1+\sqrt{\varepsilon(n-k-1)}) .
$$

Putting together the bounds (4.20), (4.22), and (4.31), we find:

$$
\begin{aligned}
M_{n}^{\varepsilon} \leqq & c\left((\sqrt{n \varepsilon}+1)\left\|\omega_{0}\right\|+\left\|u_{0}\right\|\right) \\
& +c \sum_{k=0}^{n-1} \varepsilon\left[\left(M_{k}^{\varepsilon}\right)^{2}\left(1+\sqrt{\varepsilon(n-k-1)}+M_{k}^{\varepsilon}\right)+\left(M_{k}^{\varepsilon}\right)^{2} / \sqrt{\varepsilon(n-k)}\right]
\end{aligned}
$$

which implies very easily Theorem 1 .

\section{Some Properties of the Stokes Semiflow}

Our aim in this section is to give some estimates on the Stokes semiflow which will be useful in the sequel.

Suppose $\gamma(x, y)$ is an initial profile of vorticity, not necessarily satisfying the boundary conditions. The Stokes semiflow is defined, according to Sect. 2, as

where

$$
S_{t} \gamma=G_{t}(\gamma+a \delta)+\int_{0}^{t} d s G_{t-s}\left(f_{s} \delta\right)
$$

$$
a(x)=-2 u_{0}^{(1)}\left(x, 0^{+}\right), \text {and } u_{0}=\nabla^{\perp} \Delta_{D}^{-1} \gamma .
$$

Moreover, $f$ satisfies Eq. (2.22) [or Eq. (2.26)] and has the explicit solution (2.29). 
By (2.24), integrating by parts, we have for the first and the second time derivatives of $\hat{m}$ the following expressions:

$$
\begin{gathered}
\left(\partial_{t} \hat{m}\right)(p)=2|p| \int d y g_{t}(y) \hat{\gamma}(p, y) e^{-p^{2 t}}+(2|p| / \sqrt{4 \pi t}) \hat{a}(p) e^{-p^{2} t} \\
\left(\partial_{t}^{2} \hat{m}\right)(p)=2|p| \int d y\left[\left(-p^{2}+\partial_{y}^{2}\right) g_{t}(y)\right] \hat{\gamma}(p, y) e^{-p^{2 t}}-|p| /\left(\sqrt{4 \pi t^{3 / 2}}\right) \hat{a}(p) e^{-p^{2 t}} \\
-2\left(|p|^{3} / \sqrt{4 \pi t}\right) \hat{a}(p) e^{-p^{2 t}}
\end{gathered}
$$

Lemma 5.1. For $t>s>0$, we have

$$
\left|\left(\hat{f}_{t}-\hat{f}_{s}\right)(p)\right| \leqq c|p|\left(\Gamma^{\prime}(p) \sqrt{t-s}+|\hat{a}(p)|(t-s)^{1 / 4} / s^{3 / 4}\right),
$$

where

$$
\Gamma^{\prime}(p)=\sup _{y}|\nabla \hat{\gamma}(p, y)| .
$$

Proof. By (2.22),

$$
\begin{aligned}
\left(\hat{f}_{t}-\hat{f}_{s}\right)(p)= & \left(\partial_{t} \hat{m}-\partial_{s} \hat{m}\right)(p)+p^{2}\left(\hat{m}_{t}-\hat{m}_{s}\right) \\
& +\int_{s}^{t} d \tau(|p| / \sqrt{\pi})\left(e^{-p^{2}(t-\tau)} / \sqrt{t-\tau}\right)\left(\partial_{\tau}+p^{2}\right) \hat{m}_{\tau}(p) \\
& \left.+\int_{0}^{s} d \tau(|p| / \sqrt{\pi})\left[e^{-p^{2}(t-\tau)} / \sqrt{t-\tau}\right)-\left(e^{-p^{2}(s-\tau)} / \sqrt{s-\tau}\right)\right] \\
& \times\left(\partial_{\tau}+p^{2}\right) \hat{m}_{\tau}(p) .
\end{aligned}
$$

We denote by $T_{i}, i=1, \ldots, 4$, the four terms appearing in the right-hand side of (5.7) and estimate them separately:

$$
\begin{aligned}
& \left|T_{1}\right| \leqq \int_{s}^{t} d \sigma\left|\partial_{\sigma}^{2} \hat{m}_{\sigma}(p)\right| \leqq \int_{s}^{t} d \sigma\left\{2|p|\left|\int d y\left[\left(-p^{2}+\partial_{y}^{2}\right) g_{\sigma}\right](y) \hat{\gamma}(p, y) e^{-p^{2} \sigma}\right|\right. \\
& \left.+c\left[\left(|p| / \sigma^{3 / 2}\right)+\left(|p|^{3} / \sigma^{1 / 2}\right)\right] e^{-p^{2} \sigma}|\hat{a}(p)|\right\} \\
& \leqq c|p|\left(\Gamma^{\prime}(p) \int_{s}^{t} d \sigma(1 / \sqrt{\sigma})+|\hat{a}(p)| \int_{s}^{t} d \sigma / \sigma^{3 / 2}\right) \\
& \leqq c|p|\left(\Gamma^{\prime}(p)(\sqrt{t}-\sqrt{s})+|\hat{a}(p)|[(1 / \sqrt{s})-(1 / \sqrt{t}))\right], \\
& \left|T_{2}\right| \leqq p^{2} \int_{s}^{t} d \sigma\left|\partial_{\sigma} \hat{m}(p)\right| \leqq p^{2} \int_{s}^{t} d \sigma\left\{2|p|\left|\int d y g_{\sigma}(y) \hat{\gamma}(p, y) e^{-p^{2} \sigma}\right|\right. \\
& \left.+c(|p| / \sqrt{\sigma})|\hat{a}(p)| e^{-p^{2} \sigma}\right\} \\
& \leqq c|p|\left(\Gamma^{\prime}(p)(\sqrt{t}-\sqrt{s})+|\hat{a}(p)|[(1 / \sqrt{s})-(1 / \sqrt{t}))\right] .
\end{aligned}
$$

By virtue of the estimate

we have

$$
\left|\left(\partial_{\tau}+p^{2}\right) \hat{m}_{\tau}(p)\right| \leqq c e^{-p^{2} \tau}\left(|p| \sup _{y}|\hat{\gamma}(p, y)|+|p||\hat{a}(p)| / \sqrt{\tau}\right),
$$

$$
\begin{aligned}
\left|T_{3}\right| & \leqq c \int_{s}^{t} d \tau\left(e^{-p^{2} \tau} / \sqrt{t-\tau}\right)\left(|p| \Gamma^{\prime}(p)+p^{2} \mid \hat{a}(p) / \tau\right) \\
& \leqq c \sqrt{t-s}|p| \Gamma^{\prime}(p)+c\left[\int_{s}^{t}(d \tau / \sqrt{t-\tau} \sqrt{\tau}) p^{2}|\hat{a}(p)| e^{-p^{2} t}\right] \\
& \leqq c|p|\left(\Gamma^{\prime}(p) \sqrt{t-s}+(\sqrt{t-s} / \sqrt{t s})|\hat{a}(p)|\right) .
\end{aligned}
$$


Finally, again by (5.10),

$$
\begin{aligned}
\left|T_{4}\right| \leqq & c \int_{0}^{s} d \tau|p|\left(\left(e^{-p^{2} s}-e^{-p^{2} t}\right) / \sqrt{s-\tau}\right)\left(\Gamma^{\prime}(p)+|p||\hat{a}(p)| / \sqrt{\tau}\right) \\
& +c \int_{0}^{s} d \tau|p| e^{-p^{2} t}[(1 / \sqrt{s-\tau})-(1 / \sqrt{t-\tau})]\left(\Gamma^{\prime}(p)+|p||\hat{a}(p)| / \sqrt{\tau}\right)
\end{aligned}
$$

Making use of the inequality, valid for any $\alpha \in[0,1]$,

$$
e^{-p^{2} s}-e^{-p^{2} t}=e^{-p^{2} s}\left(1-e^{-p^{2}(t-s)}\right) \leqq e^{-p^{2} s}(t-s)^{\alpha} p^{2 \alpha},
$$

we obtain for the first term in the right-hand side of (5.12) the bound

$$
\begin{aligned}
& c|p|\left(\Gamma^{\prime}(p) \sqrt{t-s}|p| e^{-p^{2} s} \int_{0}^{s} d \tau 1 / \sqrt{s-\tau}\right) \\
& \quad+|\hat{a}(p)| e^{-p^{2} s}|p|^{3 / 2}(t-s)^{1 / 4} \int_{0}^{s .} d \tau 1 /(\sqrt{s-\tau} \sqrt{\tau}) \\
& \leqq c|p|\left(\Gamma^{\prime}(p) \sqrt{t-s}+|\hat{a}(p)|(t-s)^{1 / 4} / s^{3 / 4}\right) .
\end{aligned}
$$

For the second term in the right-hand side of (5.12) we have the estimate

$$
c|p|\left(\Gamma^{\prime}(p) \sqrt{t-s}+|p| e^{-p^{2} t}|\hat{a}(p)| \int_{0}^{s} d \tau[(1 / \sqrt{s-\tau})-(1 / \sqrt{t-\tau})] / \sqrt{\tau}\right)
$$

Since

$$
\begin{aligned}
\int_{0}^{s} d \tau / \sqrt{\tau}[(1 / \sqrt{s-\tau})-(1 / \sqrt{t-\tau})] & \leqq \sqrt{t-s} \int_{0}^{s} d \tau / \sqrt{\tau(s-\tau)(t-\tau)} \\
& \leqq(t-s)^{1 / 4} \int_{0}^{s} d \tau /\left(\sqrt{\tau}(s-\tau)^{3 / 4}\right) \\
& \leqq c((t-s) / s)^{1 / 4}
\end{aligned}
$$

(5.15) turns out to be bounded by

$$
c|p|\left(\Gamma^{\prime}(p) \sqrt{t-s}+|\hat{a}(p)|(t-s)^{1 / 4} / s^{3 / 4}\right) .
$$

Collecting all the above estimates and realizing that $\sqrt{t-s} \leqq(t-s)^{1 / 4} t^{1 / 4}$, we conclude the proof of the lemma.

Lemma 5.2. Putting $\Gamma(p)=\sup _{y}|\hat{\gamma}(p, y)|$, we have the following bounds:

$$
\begin{gathered}
\left|\hat{f}_{t}(p)\right| \leqq c e^{-p^{2} t}|p|(\Gamma(p)+|\hat{a}(p)| / \sqrt{t}), \\
\left\|S_{t} \gamma\right\| \leqq c(\|\gamma\|+\|a\| / \sqrt{t}), \\
\left\|\nabla S_{t} \gamma\right\| \leqq c\left(\|\nabla \gamma\|+\|a\|^{(1)} / \sqrt{t}+\|a\| / t\right) .
\end{gathered}
$$

Proof. Estimate (5.18) is obvious after (5.10) and (2.29) and (5.19) is suddenly obtained after inserting (5.18) in (5.1). 
To obtain (5.20), consider the equality

$$
\nabla S_{t} \gamma=\nabla G_{t} \gamma+\nabla G_{t} a \delta+\int_{0}^{t} d s \nabla G_{t-s}\left(f_{s} \delta\right) .
$$

We have

$$
\begin{gathered}
\left\|\nabla G_{t} \gamma\right\| \leqq c\|\nabla \gamma\|, \\
\left\|\nabla G_{t} a \delta\right\| \leqq c \int d p e^{-p^{2 t}} \sup _{y}\left[(|p|+|y| / t) g_{t}(y)\right]|\hat{a}(p)| \leqq(c / t)\|a\|, \\
\left|\int_{0}^{t} d s \partial_{y} \widehat{G}_{t-s} f_{s} \delta(p, y)\right| \leqq c \int_{0}^{t} d s(|y| /(t-s)) g_{t-s}(y) e^{-p^{2}(t-s)}\left|\hat{f}_{s}\right| \\
\leqq c\left|\hat{f}_{t}\right| \int_{0}^{t}[d s /(t-s)]|y| g_{t-s}(y) \\
+c \int_{0}^{t}[d s /(t-s)] e^{-p^{2}(t-s)}\left|\hat{f}_{t}(p)-\hat{f}_{s}(p)\right| .
\end{gathered}
$$

The first integral in the right-hand side of (5.24) is uniformly bounded. Inserting (5.5) in the second one, we get:

$$
\left|\int_{0}^{t} d s \partial_{y} G_{t-s} f_{s} \delta(p, y)\right| \leqq c\left\{\left|\hat{f}_{t}(p)\right|+\Gamma^{\prime}(p)+|p||\hat{a}(p)| / \sqrt{t}\right\} .
$$

A similar estimate holds replacing $\partial_{y}$ by $\partial_{x}$. Therefore, by the use of (5.18), we obtain (5.20).

\section{Lemma 5.3.}

$$
\begin{gathered}
\left\|\nabla^{\perp} \Delta_{D}^{-1} S_{t} \gamma\right\| \leqq c\left(\left\|\nabla^{\perp} \Delta_{D}^{-1} \gamma\right\|+\sqrt{t}\|\gamma\|\right), \\
\left\|\nabla^{\perp} \Delta_{D}^{-1} S_{t} \partial_{i} \gamma\right\| \leqq c\|\gamma\| .
\end{gathered}
$$

Proof. Proceeding as in (4.25), putting $u_{\gamma}=\nabla^{\perp} \Delta_{D}^{-1} \gamma$, we have

$$
\begin{aligned}
G_{t}(\gamma+a \delta) & =G_{t}\left(\varepsilon_{y} \nabla^{\perp} u_{\gamma}\right)-2 G_{t}\left[u_{\gamma}^{(1)}(\cdot, 0) \delta\right]=G_{t} \nabla^{\perp}\left(\varepsilon_{y} u_{\gamma}\right) \\
& =\nabla^{\perp} G_{t}\left(\varepsilon_{y} u_{\gamma}\right) .
\end{aligned}
$$

Hence, by Eqs. (2.20) and (2.21),

$$
\left\|\nabla^{\perp} \Delta_{D}^{-1} G(\gamma+a \delta)\right\|=\left\|\nabla^{\perp} \Delta_{D}^{-1} \nabla^{\perp} G_{t}\left(\varepsilon_{y} u_{\gamma}\right)\right\| \leqq c\left\|G_{t}\left(\varepsilon_{y} u_{\gamma}\right)\right\| \leqq c\left\|u_{\gamma}\right\| .
$$

Moreover, by (5.18):

$$
\begin{aligned}
\left|\nabla^{\perp} \Delta_{D}^{-1} \int d s G_{t-s} f_{s} \delta(p, y)\right| & \leqq c \int_{0}^{t} d s e^{-p^{2}(t-s)}\left|\hat{f}_{s}(p)\right| \\
& \leqq c \sqrt{t}\{\Gamma(p)+|\hat{a}(p)| / \sqrt{t}\},
\end{aligned}
$$

and this estimate achieves the proof of (5.26).

Expression (5.27) follows easily by the inequality $\left\|\nabla^{\perp} \Delta_{D}^{-1} \partial_{i} \gamma\right\| \leqq c\|\gamma\|$ and by estimating as in (5.18) the new $f$ generated by the initial condition $\nabla \gamma$. 
Lemma 5.4. Suppose $a=0$. Then, for all $\eta \in(0,1]$,

Proof.

$$
\begin{gathered}
\left\|\left(S_{\sigma}-I\right) \gamma\right\| \leqq c\|\nabla \gamma\|^{\eta}\|\gamma\|^{1-\eta} \sigma^{\eta / 2} \\
\left\|\nabla^{\perp} \Delta_{D}^{-1}\left(S_{\sigma}-I\right) \gamma\right\| \leqq c \sqrt{\sigma}\|\gamma\| . \\
\left(G_{\sigma}-\widehat{I}\right) \gamma(p, y)=\int_{0}^{\sigma} d \tau\left(-p^{2}+\partial_{y}^{2}\right) G_{\tau}^{\hat{\gamma}} \gamma(p, y), \\
\left\|\left(G_{\sigma}-I\right) \gamma\right\| \leqq c \int_{0}^{\sigma} d \tau\left(\int(d p / \sqrt{\tau}) \sup _{y}|\nabla \hat{\gamma}(p, y)|\right)^{\eta}, \\
\left(\int(d p / \tau) \sup _{y}|\hat{\gamma}(p, y)|\right)^{1-\eta} \leqq c\|\nabla \gamma\|^{\eta}\|\gamma\|^{1-\eta} \int_{0}^{\sigma} d \tau(1 / \tau){ }^{1-\frac{\eta}{2}} \\
=c\|\nabla \eta\|^{\eta}\|\gamma\|^{1-\eta} \sigma^{\eta / 2}
\end{gathered}
$$

On the other side, by (5.18) (since $a=0)$,

$$
\int_{0}^{\sigma} d \tau\left(e^{-p^{2}(\sigma-\tau)} / \sqrt{\sigma-\tau}\right)\left|\hat{f}_{\tau}\right| \leqq c \int_{0}^{\sigma} d \tau\left(e^{-p^{2}(\sigma-\tau)} e^{-p^{2} \tau} / \sqrt{\sigma-\tau}\right) \Gamma(p)|p|
$$

Therefore, the norm of the right-hand side of (5.35) is simultaneously bounded by

and by

$$
c\|\nabla \gamma\| \sqrt{\sigma}
$$

$$
c\|\gamma\| \int_{0}^{\sigma} d \tau / \sqrt{\sigma-\tau} \sqrt{\sigma}=c\|\gamma\| .
$$

Interpolating the bounds (5.36) and (5.37), we complete the proof of (5.31).

Finally, by (5.33), (2.20), and (2.21),

$$
\begin{gathered}
\left|\nabla^{\perp} \Delta_{D}^{-1}\left(G_{\sigma}-\hat{I}\right) \gamma(p, y)\right| \leqq c \int_{0}^{\sigma}(d \tau / \sqrt{\tau}) \Gamma(p), \\
\left|\nabla^{\perp} \Delta_{D}^{-1} \int_{0}^{\sigma} d s G_{\sigma-s} f_{s} \delta(p, y)\right| \leqq c \int_{0}^{\sigma}(d s / \sqrt{\sigma-s}) \Gamma(p),
\end{gathered}
$$

which imply (5.32).

\section{Convergence of the Stokes Algorithm}

Consider Eq. (2.26) associated to an initial vorticity profile and its discrete version

$$
\hat{h}_{m}^{\varepsilon}=\left[\left(I-T_{\varepsilon}\right) \hat{f}^{\varepsilon}\right]_{m} .
$$

It will be convenient to think of Eq. (6.1) as defined for all times $t \geqq 0$ by considering $\hat{h}^{\varepsilon}$ and $\hat{f}^{\varepsilon}$ as functions defined on $\mathbb{R}^{+}$by

$$
\begin{aligned}
& \hat{h}_{s}^{\varepsilon}=\hat{h}_{s_{\varepsilon}}^{\varepsilon}, \quad \hat{f}_{s}^{\varepsilon}=\hat{f}_{s_{\varepsilon}}^{\varepsilon}, \quad S_{\varepsilon}=[s / \varepsilon] \varepsilon, \quad s \geqq \varepsilon, \\
& \hat{h}_{s}^{\varepsilon}=\hat{f}_{s}^{\varepsilon}=\hat{h}_{1}^{\varepsilon}, \quad 0 \leqq s<\varepsilon,
\end{aligned}
$$


and $T_{\varepsilon}$ extended on arbitrary functions by

$$
\begin{aligned}
& \left(T_{\varepsilon} l\right)_{t}=\int_{\varepsilon}^{t_{\varepsilon}} d s(|p| / \sqrt{\pi})\left(e^{-p^{2}\left(t_{\varepsilon}-s\right)} / \sqrt{t_{\varepsilon}-s}\right) l_{s_{\varepsilon}}, \quad t \geqq \varepsilon, \\
& \left(T_{\varepsilon} l_{t}=0, \quad 0 \leqq t<\varepsilon .\right.
\end{aligned}
$$

With these conventions we take the difference between Eqs. (2.26) and (6.1):

$$
\left(I-T_{\varepsilon}\right) \xi^{\varepsilon}=\left(T_{\varepsilon}-T\right) \hat{f}+\left(\hat{h}^{\varepsilon}-\hat{h}\right),
$$

where

$$
\hat{\xi}^{\varepsilon}=\hat{f}^{\varepsilon}-\hat{f} .
$$

To estimate $\hat{\xi}$ we need two preparatory lemmas.

Lemma 6.1. For $t=n \varepsilon, n \geqq 1$,

$$
\left|\left[\left(T_{\varepsilon}-T\right) \hat{f}\right]_{t}\right|(p) \leqq c \sqrt{\varepsilon}|p| \Gamma^{\prime}(p)+c|p|\left(\varepsilon / t^{3}\right)^{1 / 4}|\hat{a}(p)| .
$$

Proof. We have, for $n>1$ :

$$
\begin{aligned}
& {\left[\left(T-T_{\varepsilon}\right) \hat{f}\right]_{t}(p)} \\
& \quad=(|p| / \sqrt{\pi})\left\{\int_{\varepsilon}^{t} d s\left(e^{-p^{2}(t-s)} / \sqrt{t-s}\right)\left(\hat{f}_{s}-\hat{f}_{s_{\varepsilon}}\right)(p)+\int_{0}^{\varepsilon} d s\left(e^{-p^{2}(t-s)} / \sqrt{t-s}\right) \hat{f}_{s}(p)\right\} .
\end{aligned}
$$

By Lemma 5.1 and estimate (5.18):

$$
\begin{aligned}
\left|\left[\left(T-T_{\varepsilon}\right) \hat{f}\right]_{t}\right|(p) \leqq & c p^{2} \int_{\varepsilon}^{t} d s\left\{\Gamma^{\prime}(p)(\sqrt{\varepsilon} / \sqrt{t-s})+|\hat{a}(p)| \varepsilon^{1 / 4} /\left(\sqrt{t-s s_{\varepsilon}^{3 / 4}}\right)\right\} e^{-p^{2}(t-s)} \\
& +c p^{2} \Gamma(p) \int_{0}^{\varepsilon} d s / \sqrt{t-s}+c p^{2} e^{-p^{2} t}|\hat{a}(p)| \int_{0}^{\varepsilon} d s / \sqrt{(t-s) s} \\
\leqq & c\left(|p| \Gamma^{\prime}(p) \sqrt{\varepsilon}+|p||\hat{a}(p)|\left(\varepsilon / t^{3}\right)^{1 / 4}\right) \\
& +c p^{2} \Gamma(p) \sqrt{\varepsilon}+c p^{2}(\sqrt{\varepsilon} / \sqrt{t-\varepsilon})|\hat{a}(p)| e^{-p^{2} t}
\end{aligned}
$$

On the other hand

$$
\left[\left(T-T_{\varepsilon}\right) \hat{f}\right]_{\varepsilon}(p) \leqq c p^{2} e^{-p^{2} \varepsilon}(|\hat{a}(p)|+\sqrt{\varepsilon} \Gamma(p)),
$$

and this achieves the proof.

Lemma 6.2. For $t=n \varepsilon$ and $n \geqq 1$,

$$
\left|\widehat{h}_{n}^{\varepsilon}-\widehat{h}_{n \varepsilon}\right| \leqq c\left(\sqrt{\varepsilon}|p| \Gamma^{\prime}(p)+\left(\varepsilon / t^{3}\right)^{1 / 2}|p||\hat{a}(p)|\right) .
$$

Proof. Let $n>1$; recalling Eq. (5.4):

$$
\begin{aligned}
\left|\hat{h}_{t}^{\varepsilon}-\hat{h}_{t}\right| & =\left|(D \hat{m})_{t}-(\partial \hat{m})_{t}\right|=\varepsilon^{-1}\left|\int_{t-\varepsilon}^{t} d \sigma\left(\partial_{\sigma} \hat{m}-\partial_{t} \hat{m}\right)\right|=\varepsilon^{-1}\left|\int_{t-\varepsilon}^{t} d \sigma \int_{\sigma}^{t} d \tau \partial_{\tau}^{2} \hat{m}_{\tau}\right| \\
& \leqq c \varepsilon^{-1}|p||\hat{a}(p)| \int_{t-\varepsilon}^{t} d \sigma \int_{\sigma}^{t} d \tau 1 / \tau^{3 / 2}+c \varepsilon^{-1}|p| \Gamma^{\prime}(p) \int_{t-\varepsilon}^{t} d \sigma \int_{\sigma}^{t} d \tau 1 / \sqrt{\tau} \\
& \left.\leqq c \varepsilon^{1 / 4} /(t-\varepsilon)^{3 / 2}\right)|p||\hat{a}(p)|+c \varepsilon|p| \Gamma^{\prime}(p) / \sqrt{t-\varepsilon} \\
& \leqq c \varepsilon^{1 / 4}|p||\hat{a}(p)| /(t-\varepsilon)^{3 / 4}+c \sqrt{\varepsilon}|p| \Gamma^{\prime}(p)
\end{aligned}
$$


For $n=1$ :

$$
\begin{aligned}
\left|\hat{h}_{\varepsilon}^{\varepsilon}-\hat{h}_{\varepsilon}\right|(p) & \leqq \varepsilon^{-1} \int_{0}^{\varepsilon} d \sigma \int_{0}^{t} d \tau 2|p|\left|\int d y\left(-p^{2}+\partial_{y}^{2}\right) g_{t}(y) \hat{\gamma}(p, y) e^{-p^{2} \tau}\right| \\
& +c \varepsilon^{-1} \int_{0}^{\varepsilon} d \sigma(|p| / \sqrt{\sigma})|\hat{a}(p)| \leqq c\left(\sqrt{\varepsilon}|p| \Gamma^{\prime}(p)+|p||\hat{a}(p)| / \sqrt{\varepsilon}\right)
\end{aligned}
$$

Hence (6.10) is proved.

Lemma 6.3. For $t=n$ and $n \geqq 1$ :

$$
\left|\hat{\xi}_{t}^{\varepsilon}(p)\right| \leqq c \sqrt{\varepsilon}|p|\left(1+p^{2} t\right) \Gamma^{\prime}(p)+c \varepsilon^{1 / 4}\left(|p| / t^{3 / 4}\right)\left(p^{2} t+1\right)|\hat{a}(p)| .
$$

Proof. By Eqs. (6.4) and (2.31)

where

$$
\hat{\xi}_{n \varepsilon}^{\varepsilon}=l_{n}+\left(T_{\varepsilon} l_{n}+R_{n},\right.
$$

$$
l_{n}=\left[\left(T_{\varepsilon}-T\right) \hat{f}\right]_{n}+\left(\hat{h}^{\varepsilon}-\hat{h}\right)_{n}
$$

and

$$
R_{n}=\sum_{k=2}^{n-1}\left(T_{\varepsilon}^{k} l\right)_{n}
$$

By (2.35), Proposition 1, Lemmas 6.1 and 6.2

$$
\begin{aligned}
\left|R_{n}\right| & \leqq c \lambda \sum_{m=1}^{n-2}\left|l_{m}\right| \leqq c\left\{\sqrt{\varepsilon}|p|^{3} \Gamma^{\prime}(p) t+|p||\hat{a}(p)| \lambda \sum_{m=1}^{n-2}\left(\varepsilon^{1 / 4} / \varepsilon^{3 / 4}\right) m^{-3 / 4}\right\} \\
& \leqq c\left\{\sqrt{\varepsilon}|p|^{3} t \Gamma^{\prime}(p)+\sqrt{\varepsilon}|p|^{3}|\hat{a}(p)| n^{1 / 4}\right\} .
\end{aligned}
$$

Moreover,

$$
\begin{aligned}
\left|\left(T_{\varepsilon} l\right)_{n}(p)\right| & \leqq c p^{2} \int_{\varepsilon}^{t} d s\left(e^{-p^{2}(t-s)} / \sqrt{t-s}\right)\left(\sqrt{\varepsilon} \Gamma^{\prime}(p)+\left(\varepsilon^{1 / 4} / s_{\varepsilon}^{3 / 4}\right)|\hat{a}(p)|\right) \\
& \leqq c|p| \sqrt{\varepsilon} \Gamma^{\prime}(p)+c\left(\varepsilon^{1 / 4} / t^{3 / 4}\right)|p||\hat{a}(p)| .
\end{aligned}
$$

This, together with estimate (6.17) and Lemmas 6.1 and 6.2, concludes the proof of Lemma 6.3.

Proof of Theorem 2. By (2.14) and (2.18), for $t=n$,

$$
\begin{aligned}
\left(S_{t}-S_{n}^{\varepsilon}\right) \gamma= & -\sum_{k=1}^{n-1} \varepsilon G_{(n-k) \varepsilon} \xi_{k \varepsilon}^{\varepsilon} \delta+\int_{\varepsilon}^{t} d s\left(G_{t-s}-G_{t-s_{\varepsilon}}\right) f_{s_{\varepsilon}} \delta \\
& +\int_{0}^{\varepsilon} d s G_{t-s} f_{s} \delta+\int_{\varepsilon}^{t} d s G_{t-s}\left(f_{s}-f_{s_{\varepsilon}}\right) \delta .
\end{aligned}
$$

Denoting by $W_{i}, i=1, \ldots, 4$, the four terms appearing in the right-hand side of (6.19), we have, by Lemma 6.3:

$$
\begin{gathered}
\left|\hat{W}_{1}(p, y)\right| \leqq c \sum_{k=1}^{n-1} \varepsilon\left(e^{-p^{2}(n-k) \varepsilon} / \sqrt{(n-k) \varepsilon}\right)\left|\hat{\xi}_{k \varepsilon}^{\varepsilon}(p)\right| \\
\left\|W_{1}\right\| \leqq c \sqrt{\varepsilon}\left(\|\gamma\|^{(3,0)}+\|\gamma\|^{(2,1)}\right)(1+t)+c(\varepsilon / t)^{1 / 4}(1+t)\|a\|^{(2)},
\end{gathered}
$$


since

$$
|p| \sum_{k=1}^{n-1} \varepsilon e^{-p^{2}(n-k) \varepsilon} / \sqrt{(n-k) \varepsilon} \leqq c, \quad \sum_{k=1}^{n-1} \varepsilon e^{-p^{2}(n-k) \varepsilon} /\left(\sqrt{(n-k) \varepsilon(k \varepsilon)^{3 / 2}}\right) \leqq c / t^{1 / 4} .
$$

Furthermore,

$$
\begin{aligned}
&\left|\hat{W}_{2}(p, y)\right| \leqq \int_{\varepsilon}^{t} d s\left|\left(G_{s-s_{\varepsilon}}-I\right) \widehat{G}_{t-s} f_{s_{\varepsilon}} \delta\right|(p, y) \\
& \leqq\left.\int_{\varepsilon}^{t} d s\left(\int_{0}^{s-s_{\varepsilon}} d \tau \mid \partial_{\tau} G_{t+\tau-s} \widehat{\left(f s_{s_{\varepsilon}}\right.} \delta\right)(p, y) \mid\right)^{1 / 2} \\
& \times\left(\sup _{y}\left[\left|G_{t-s} \widehat{f_{s_{\varepsilon}}} \delta\right|+\left|G_{t-s_{\varepsilon}} \widehat{f_{s_{\varepsilon}}} \delta\right|\right]\right)^{1 / 2} \\
& \leqq\left.c \int_{\varepsilon}^{t} d s\left(\sqrt{\varepsilon} \sup _{y} \mid \nabla G_{t-s} \widehat{\left(f_{s_{\varepsilon}}\right.} \delta\right) \mid\right)^{1 / 2}\left|\hat{f}_{s_{\varepsilon}}(p)\right|^{1 / 2} /(t-s)^{1 / 4} \\
& \leqq c \varepsilon^{1 / 4} \int_{0}^{t} d s(t-s)^{-3 / 4}\left|\hat{f}_{s_{\varepsilon}}(p)\right| \\
&\left\|W_{2}\right\| \leqq c(\varepsilon t)^{1 / 4}\|\gamma\|^{(1,0)}+c(\varepsilon / t)^{1 / 4}\|a\|^{(1)} \\
&\left|\hat{W}_{3}(p, y)\right| \leqq c \int_{0}^{\varepsilon} d s(1 / \sqrt{t-s})|p|(\Gamma(p)+|\hat{a}(p)| / \sqrt{s}) \\
&\left\|W_{3}\right\| \leqq c\left(\sqrt{\varepsilon}\|\gamma\|^{(1,0)}+\sqrt{\varepsilon / t}\|a\|^{(1)}\right) .
\end{aligned}
$$

By Lemma 5.1

$$
\left|\hat{W}_{4}(p, y)\right| \leqq c|p| \int_{\varepsilon}^{t} d s\left\{\Gamma^{\prime}(p) \sqrt{\varepsilon} / \sqrt{t-s}+|\hat{a}(p)| \varepsilon^{1 / 4} /\left(\sqrt{t-s} s_{\varepsilon}^{3 / 4}\right)\right\} e^{-p^{2}(t-s)} .
$$

Whence

$$
\left.\left\|W_{4}\right\| \leqq c \sqrt{\varepsilon t}\left(\|\gamma\|^{(2,0)}+\|\gamma\|^{(1,1)}\right)+(\varepsilon / t)^{1 / 4}\|a\|^{(1)}\right)
$$

This proves the first part of Theorem 2 .

Finally, again by (2.14) and (2.18),

$$
\begin{aligned}
\nabla^{\perp} \Delta_{D}^{-1}\left(S_{n+1}^{\varepsilon}-S_{n \varepsilon}\right) \gamma(p, y)= & \nabla^{\perp} \Delta_{D}^{-1}\left\{\left(G_{\varepsilon}-I\right) G_{t}(\gamma+a \delta)\right\}+\sum_{k=1}^{n} \varepsilon G_{(n+1-k) \varepsilon} \xi_{k \varepsilon}^{\varepsilon} \delta \\
& +\int_{0}^{t} d s\left(G_{t-s_{\varepsilon}}-G_{t-s}\right) f_{s_{\varepsilon}+\varepsilon} \delta+\int_{0}^{t} d s G_{t-s}\left(f_{s_{\varepsilon}+\varepsilon}-f_{s_{\varepsilon}}\right) \delta .
\end{aligned}
$$

The first term in the right-hand side of (6.29), using (2.20), (2.21), and some integrations by parts, can be bounded by

$$
c\left(\sqrt{\varepsilon} \Gamma(p)+\int_{0}^{\varepsilon}(d \sigma / \sqrt{\sigma})|p||\hat{a}(p)|\right) .
$$


Therefore, proceeding as in the estimate leading to (2.52), we have:

$$
\begin{aligned}
\left\|\nabla^{\perp} \Delta_{D}^{-1}\left(S_{n+1}^{\varepsilon}-S_{n \varepsilon}\right) \gamma\right\| \leqq & c\left(\sqrt{\varepsilon}\|\gamma\|+\sqrt{\varepsilon}\|a\|^{(1)}\right) \\
& +\left[c \sqrt{\varepsilon}(1+t)\left(\|\gamma\|^{(2,0)}+\|\gamma\|^{(1,1)}\right)+c(\varepsilon / t)^{1 / 4}(1+t)\|a\|^{(1)}\right] \\
& +c(\varepsilon t)^{1 / 4}\|\gamma\|+c(\varepsilon / t)^{1 / 4}\|a\|+c \sqrt{\varepsilon t}\left(\|\gamma\|^{(1,0)}+\|\gamma\|^{(0,1)}\right) \\
& +c(\varepsilon / t)^{1 / 4}\|a\| .
\end{aligned}
$$

In a completely analogous way, using decomposition (6.19), we have:

$$
\begin{aligned}
\left\|\nabla^{\perp} \Delta_{D}^{-1}\left(S_{t}-S_{n}^{\varepsilon}\right) \gamma\right\| \leqq & c(1+t)\left[\sqrt{\varepsilon}\left(\|\gamma\|^{(2,0)}+\|\gamma\|^{(1,1)}\right)+(\varepsilon / t)^{1 / 4}\|a\|^{(1)}\right] \\
& +c\left[(\varepsilon / t)^{1 / 4}\|\gamma\|+(\varepsilon / t)^{1 / 4}\|a\|\right] \\
& +c\left[\sqrt{\varepsilon}\|\gamma\|+\sqrt{\varepsilon}\|a\|^{(1)}\right] \\
& \left.+c\left[\sqrt{\varepsilon / t}\|\gamma\|^{(1,0)}+\|\gamma\|^{(0,1)}\right)+(\varepsilon / t)^{1 / 4}\|a\|\right] .
\end{aligned}
$$

This concludes the proof of Theorem 2.

\section{Proof of Theorem 3}

We consider a time interval $[0, T]$, with $T$ to be fixed later. Our starting point is the expansion (2.54). We denote by $\alpha_{i}, i=0, \ldots, 4$, the five terms appearing in the righthand side of (2.54) and estimate each of them separately.

$$
\begin{aligned}
\left\|\alpha_{0}\right\| & \leqq\left\|\left(S_{n \varepsilon}-S_{n}^{\varepsilon}\right) \omega_{0}\right\|+\left\|S_{n}^{\varepsilon}\left(I-G_{\varepsilon}\right) \omega_{0}\right\| \\
& \leqq c(1+t)\left\|\omega_{0}\right\|^{(3,1)} \varepsilon^{1 / 4}+c\left\|\left(I-G_{\varepsilon}\right) \omega_{0}\right\|+(c / \sqrt{n \varepsilon})\left\|\nabla^{\perp} \Delta_{D}^{-1}\left(I-G_{\varepsilon}\right) \omega_{0}\right\|
\end{aligned}
$$

by virtue of Theorem 2 and Proposition 3 [recalling that $\nabla^{\perp} \Delta_{D}^{-1} \omega_{0}\left(x, 0^{+}\right)=0$ ].

It is easily seen that the last two terms in the right-hand side of (7.1) can be bounded by $c \sqrt{\varepsilon}\left\|\omega_{0}\right\|^{(1,1)}$ and by $(c \varepsilon / \sqrt{\varepsilon n})\left\|\omega_{0}\right\|^{(1,1)}$, respectively. Therefore, for $\varepsilon$ small,

$$
\left\|\alpha_{0}\right\| \leqq c(1+t) \varepsilon^{1 / 4}\left\|\omega_{0}\right\|^{(3,1)} .
$$

Furthermore, $\alpha_{1}=\beta_{1}+\beta_{2}$, where

$$
\begin{gathered}
\beta_{1}=\int_{0}^{t} d s\left(S_{t-s}-S_{t-s_{\varepsilon}}\right)\left[\left(u_{s_{\varepsilon}} \cdot \nabla\right) \omega_{s_{\varepsilon}}\right] \\
\beta_{2}=\int_{0}^{t} d s S_{t-s}\left[\left(u_{s} \cdot \nabla\right) \omega_{s}-\left(u_{s_{\varepsilon}} \cdot \nabla\right) \omega_{s_{\varepsilon}}\right] .
\end{gathered}
$$

Then, by Lemma 5.4, (5.19) and (5.20),

$$
\begin{aligned}
\left\|\beta_{1}\right\| & \leqq c \varepsilon^{1 / 4} \int_{0}^{t} d s\left\|\nabla S_{t-s}\left(u_{s_{\varepsilon}} \cdot \nabla\right) \omega_{s_{\varepsilon}}\right\|^{1 / 2}\left\|S_{t-s}\left(u_{s_{\varepsilon}} \cdot \nabla\right) \omega_{s_{\varepsilon}}\right\|^{1 / 2} \\
& =c(T) \varepsilon^{1 / 4} \int_{0}^{t} d s\left(\left\|\left(u_{s_{\varepsilon}} \cdot \nabla\right) \omega_{s_{\varepsilon}}\right\|^{(1,1)} /(t-s)^{1 / 4}+\left\|\tilde{a}_{s_{\varepsilon}}\right\|^{(1)} /(t-s)^{3 / 4}\right)
\end{aligned}
$$


where $\tilde{a}_{S_{\varepsilon}}$ is the velocity field generated, on the boundary, by $\left(u_{s_{\varepsilon}} \cdot \nabla\right) \omega_{s_{\varepsilon}}$. Furthermore,

$$
\begin{aligned}
\left\|\left(u_{s_{\varepsilon}} \cdot \nabla\right) \omega_{s_{\varepsilon}}\right\|^{(1,1)} & \leqq c\left\|u_{s_{\varepsilon}}||^{(1,1)}\right\| \omega_{s_{\varepsilon}} \|^{(2,2)} \\
\left\|\tilde{a}_{s_{\varepsilon}}\right\|^{(1)} & \leqq \int d p \mid \int_{0}^{\infty} d y e^{-|p| y|p|\left|u_{s_{\varepsilon}} \cdot \nabla \omega_{s_{\varepsilon}}\right|} \leqq c\left\|u_{s_{\varepsilon}}\right\|\left\|\omega_{s_{\varepsilon}}\right\|^{(1,1)} .
\end{aligned}
$$

Therefore,

$$
\left\|\beta_{1}\right\| \leqq c\left(\omega_{0}, T\right) \varepsilon^{1 / 4}
$$

by the use of Theorem 4.1 of [6], for which $\left\|u_{s}\right\|^{(1,1)}$ and $\left\|\omega_{s}\right\|^{(1,1)}$ are bounded functions of the time, while $\left\|\omega_{s}\right\|^{(2,2)} \leqq c\left(\omega_{0}\right) / \sqrt{s}$ provided that $\left\|\omega_{0}\right\|^{(4,2)}<+\infty$.

We have also

where

$$
\beta_{2}=\beta_{2}^{1}+\beta_{2}^{2},
$$

$$
\beta_{2}^{1}=\int_{0}^{t} d s S_{t-s}\left(u_{s}-u_{s_{\varepsilon}}\right) \cdot \nabla \omega_{s}
$$

and

$$
\beta_{2}^{2}=\int_{0}^{t} d s S_{t-s}\left(u_{s_{\varepsilon}} \cdot \nabla\right)\left(\omega_{s}-\omega_{s_{\varepsilon}}\right)
$$

satisfy the bounds

$$
\begin{gathered}
\left\|\beta_{2}^{1}\right\| \leqq c \int_{0}^{t} d s\left\{\left\|u_{s}-u_{s_{\varepsilon}}\right\|\left\|\nabla \omega_{s}\right\|+\left\|u_{s}-u_{s_{\varepsilon}}\right\|\left\|\omega_{s}\right\| / \sqrt{t-s}\right\} \\
\leqq \varepsilon c\left(\omega_{0}, T\right) \int_{0}^{t} d s\left\{\left\|\nabla \omega_{s}\right\|+\left\|\omega_{s}\right\| / \sqrt{t-s}\right\} \\
\left\|\beta_{2}^{2}\right\| \leqq c \int_{0}^{t} d s\left\|\omega_{s}-\omega_{s_{\varepsilon}}\right\|\left\{\left\|\omega_{s_{\varepsilon}}\right\|+\left\|u_{s_{\varepsilon}}\right\| / \sqrt{t-s}\right\} \\
\leqq \sqrt{\varepsilon} c\left(\omega_{0}, T\right) \int_{0}^{t} d s\left(\left\|\omega_{s_{\varepsilon}}\right\|+\left\|u_{s_{\varepsilon}}\right\| / \sqrt{t-s}\right) .
\end{gathered}
$$

For the bound (7.11) we have used Lemma 5.2, the obvious bound

$$
\left\|\nabla^{\perp} \Delta_{D}^{-1}\left(u_{s}-u_{s_{\varepsilon}}\right) \cdot \nabla \omega_{s}\right\|=\left\|\nabla^{\perp} \Delta_{D}^{-1} \nabla \cdot\left(u_{s}-u_{s_{\varepsilon}}\right) \omega_{s}\right\| \leqq c\left\|u_{s}-u_{s_{\varepsilon}}\right\|\left\|\omega_{s}\right\|,
$$

and the estimate (see $[6$, Theorem 4.1])

$$
\left\|\partial_{t} u_{t}\right\| \leqq c\left(\omega_{0}, T\right) .
$$

For the bound (7.12) we have used the estimate (2.47) of Proposition 3 with $S_{n}^{\varepsilon}$ replaced by $S_{t}$ (and $\left.H=\left|\omega_{s_{\varepsilon}}\right|\right)$, the bound

$$
\left\|\nabla^{\perp} \Delta_{D}^{-1}\left(u_{s_{\varepsilon}} \cdot \nabla\right)\left(\omega_{s}-\omega_{s_{\varepsilon}}\right)\right\| \leqq c\left\|u_{s_{\varepsilon}}\right\|\left\|\omega_{s}-\omega_{s_{\varepsilon}}\right\|,
$$

and the estimate (see [6, Theorem 4.1])

$$
\left\|\partial_{t} \omega_{t}\right\| \leqq c\left(\omega_{0}, T\right) / \sqrt{t} .
$$


Hence

$$
\left\|\beta_{2}\right\| \leqq \sqrt{\varepsilon} c\left(\omega_{0}, T\right)
$$

and

$$
\left\|\alpha_{1}\right\| \leqq \varepsilon^{1 / 4} c\left(\omega_{0}, T\right)
$$

By Theorem 2

$$
\begin{aligned}
\left\|\alpha_{2}\right\| & \leqq c(T) \varepsilon^{1 / 4} \sum_{k=0}^{n-1} \varepsilon\left[\left\|\left(u_{k \varepsilon} \cdot \nabla\right) \omega_{k \varepsilon}\right\|^{(3,1)}+\left\|u_{k \varepsilon} \omega_{k \varepsilon}\right\|^{(2,0) /}[(n-k) \varepsilon]^{1 / 4}\right] \\
& \leqq \varepsilon^{1 / 4} c(T) \sum_{k=0}^{n-1} \varepsilon\|u\|^{(3,1)}\left\|\omega_{k \varepsilon}\right\|^{(4,2)} /[(n-k) \varepsilon]^{1 / 4} .
\end{aligned}
$$

By Theorem 4.1 of ref [6] it is easy to argue that $\left\|u_{t}\right\|^{(3,1)}$ is bounded and

$$
\left\|\omega_{t}\right\|^{(4,2)} \leqq c\left(\omega_{0}, T\right) / \sqrt{t}
$$

provided that $\left\|\omega_{0}\right\|^{(6,2)}<+\infty$. Hence

$$
\left\|\alpha_{2}\right\| \leqq \varepsilon^{1 / 4} c\left(\omega_{0}, T\right)
$$

By Proposition 3, Eq. (2.44),

$$
\left\|\alpha_{3}\right\| \leqq \sum_{k=0}^{n-1} \varepsilon\left\{\left(\left\|u_{k \varepsilon}-b_{k}^{\varepsilon}\right\| \cdot\left\|\omega_{k \varepsilon}\right\| / \sqrt{(n-k) \varepsilon}\right)+\left\|\left(u_{k \varepsilon}-b_{k}^{\varepsilon}\right) \omega_{k \varepsilon}\right\|^{(1,0)}\right\}
$$

Since

$$
\left\|\left(u_{k \varepsilon}-b_{k}^{\varepsilon}\right) \omega_{k \varepsilon}\right\|^{(1,0)} \leqq\left\|\omega_{k \varepsilon}-S_{1}^{\varepsilon} \omega_{k}^{\varepsilon}\right\|\left\|\omega_{k \varepsilon}\right\|+\left\|u_{k \varepsilon}-b_{k}^{\varepsilon}\right\|\left\|\omega_{k \varepsilon}\right\|^{(1,0)},
$$

we conclude that

$$
\left.\left\|\alpha_{3}\right\| \leqq c\left(\omega_{0}, T\right) \sum_{k=0}^{n-1} \varepsilon\left(\left\|u_{k \varepsilon}-b_{k}^{\varepsilon}\right\|+\left\|\omega_{k \varepsilon}-S_{1}^{\varepsilon} \omega_{k}^{\varepsilon}\right\|\right) / \sqrt{(n-k) \varepsilon}\right) .
$$

Finally, by Lemma 1 and Proposition 3, since $\nabla^{\perp} b_{k}^{\varepsilon}=S_{1}^{\varepsilon} \omega_{k}^{\varepsilon}$,

$$
\left\|\alpha_{4}\right\| \leqq \sum_{k=0}^{n-1} \varepsilon\left\|\omega_{k \varepsilon}-S_{1}^{\varepsilon} \omega_{k}^{\varepsilon}\right\|\left[\left(\left\|b_{k}^{\varepsilon}\right\| / \sqrt{(n-k) \varepsilon}\right)+\left(\left\|\omega_{k}^{\varepsilon}\right\|+S_{1}^{\varepsilon} \omega_{k}^{\varepsilon}\right)\right] .
$$

Collecting all the above estimates, we obtain:

$$
\left\|\omega_{n \varepsilon}-S_{1}^{\varepsilon} \omega_{n}^{\varepsilon}\right\| \leqq c\left(\omega_{0}, T\right)\left(\varepsilon^{1 / 4}+\sum_{k=0}^{n-1} \varepsilon \eta^{\varepsilon}(k \varepsilon) / \sqrt{(n-k) \varepsilon}\right)
$$

for $T$ smaller than any time for which the stability result of Theorem 1 holds. In (7.26) we have used the notation

$$
\eta^{\varepsilon}(n \varepsilon)=\max \left\{\left\|\omega_{n \varepsilon}-S_{1}^{\varepsilon} \omega_{n}^{\varepsilon}\right\|,\left\|u_{n \varepsilon}-b_{n}^{\varepsilon}\right\|\right\} .
$$

Therefore, we need an analogous estimate for $\left\|u_{n \varepsilon}-b_{n}^{\varepsilon}\right\|$. Applying to both sides of (2.52), we obtain:

$$
u_{n \varepsilon}-b_{n}^{\varepsilon}=\sum_{i=0}^{4} \nabla^{\perp} \Delta_{D}^{-1} \alpha_{i}
$$


According to Theorem 2:

$$
\left\|\nabla^{\perp} \Delta_{D}^{-1} \alpha_{0}\right\|=\left\|\nabla^{\perp} \Delta_{D}^{-1}\left(S_{n \varepsilon}-S_{n+1}^{\varepsilon}\right) \omega_{0}\right\| \leqq \varepsilon^{1 / 4} c(T)\left\|\omega_{0}\right\|^{(3,1)} .
$$

Moreover,

$$
\nabla^{\perp} \Delta_{D}^{-1} \alpha_{1}=\nabla^{\perp} \Delta_{D}^{-1}\left(\beta_{1}+\beta_{2}^{1}+\beta_{2}^{2}\right)
$$

and, by Lemma 5.4 and Proposition 3

$$
\begin{aligned}
\left\|\nabla^{\perp} \Delta_{D}^{-1} \beta_{1}\right\| & \leqq c \sqrt{\varepsilon} \int_{0}^{t} d s\left\|S_{t-s}\left(u_{s_{\varepsilon}} \cdot \nabla \omega_{s_{\varepsilon}}\right)\right\| \\
& \left.\leqq c \sqrt{\varepsilon} \int_{0}^{t} d s\left[(1 / \sqrt{t-s})\left\|u_{s_{\varepsilon}}\right\|\left\|\omega_{s_{\varepsilon}}\right\|\right]+\left\|\omega_{s}\right\|^{2}\right] \leqq c \sqrt{\varepsilon} c\left(\omega_{0}, T\right) .
\end{aligned}
$$

By Lemma 5.3 and the bound (7.14),

$$
\left\|\nabla^{\perp} \Delta_{D}^{-1} \beta_{2}^{1}\right\| \leqq c \int_{0}^{t} d s\left\|u_{s}-u_{s_{\varepsilon}}\right\|\left\|\omega_{s}\right\| \leqq c\left(\omega_{0}, T\right) \varepsilon
$$

By the same argument, using (7.16), we have:

$$
\left\|\nabla^{\perp} \Delta_{D}^{-1} \beta_{2}^{2}\right\| \leqq c \int_{0}^{t} d s\left\|u_{s_{\varepsilon}}\right\|\left\|\omega_{s}-\omega_{s_{\varepsilon}}\right\| \leqq c\left(\omega_{0}, T\right) \sqrt{\varepsilon} .
$$

By Theorem 2

$$
\left\|\nabla^{\perp} \Delta_{D}^{-1} \alpha_{2}\right\| \leqq c(T) \varepsilon^{1 / 4} \sum_{k=0}^{n-1} \varepsilon\left(\left\|\left(u_{k \varepsilon} \cdot \nabla\right) \omega_{k \varepsilon}\right\|^{(2,1)}+\|\tilde{a}\|^{(1)} /[(n-k) \varepsilon]^{1 / 4}\right) \leqq c\left(\omega_{0}, T\right) \varepsilon^{1 / 4}
$$

By virtue of (2.45),

$$
\begin{gathered}
\left\|\nabla^{\perp} \Delta_{D}^{-1} \alpha_{3}\right\| \leqq c \sum_{k=0}^{n-1} \varepsilon\left\|u_{k \varepsilon}-b_{k}^{\varepsilon}\right\| \cdot\left\|\omega_{k \varepsilon}\right\| \leqq c\left(\omega_{0}, T\right) \sum_{k=0}^{n-1} \varepsilon\left\|u_{k \varepsilon}-b_{k}^{\varepsilon}\right\|, \\
\left\|\nabla^{\perp} \Delta_{D}^{-1} \alpha_{4}\right\| \leqq c \sum_{k=0}^{n-1} \varepsilon\left\|b_{k}^{\varepsilon}\right\|\left\|\omega_{k \varepsilon}-S_{1}^{\varepsilon} \omega_{k}^{\varepsilon}\right\| \leqq c\left(\omega_{0}, T\right) \sum_{k=0}^{n-1} \varepsilon\left\|\omega_{k \varepsilon}-S_{1}^{\varepsilon} \omega_{k}^{\varepsilon}\right\| .
\end{gathered}
$$

In the last bound we assume that $T$ is so small that the stability result of Theorem 1 holds.

Therefore,

$$
\left\|u_{n \varepsilon}-b_{n}^{\varepsilon}\right\| \leqq c\left(\omega_{0}, T\right)\left(\varepsilon^{1 / 4}+\sum_{k=0}^{n-1} \varepsilon \eta^{\varepsilon}(k \varepsilon)\right) .
$$

Combining (7.26) and (7.37), for $T$ small enough, we conclude that

$$
\eta^{\varepsilon}(n \varepsilon) \leqq c\left(\omega_{0}, T\right)\left(\varepsilon^{1 / 4}+\sum_{k=0}^{n-1} \varepsilon \eta^{\varepsilon}(k \varepsilon) / \sqrt{(n-k) \varepsilon}\right),
$$

implying

$$
\lim _{n \rightarrow \infty} \eta^{\varepsilon}(n \varepsilon)=0 \text { for } \quad n \varepsilon \leqq T
$$


To extend the validity of (7.38) to arbitrary times, we observe that, by (4.31), there exists a positive decreasing function $\delta\left(\left\|\omega_{0}\right\|,\left\|u_{0}\right\|\right)$ for which the stability result holds if $n \varepsilon<\delta\left(\left\|\omega_{0}\right\|,\left\|u_{0}\right\|\right)$. Choosing, for any fixed $\bar{T}$,

$$
\bar{\delta}=\min \left(\delta\left(2 \sup _{t \leqq T}\left\|u_{t}\right\|, 2 \sup _{t \leqq T}\left\|\omega_{t}\right\|\right), 1\right),
$$

we divide $[0, T]$ in intervals $\left[t_{i}, t_{i+1}\right]_{i=0}^{l}$ such that $\left|t_{i+1}-t_{i}\right|<\bar{\delta}$. In the first interval we obviously have convergence. Then, for $\varepsilon$ sufficiently small, we have

$$
\left\|b_{t_{1}}^{\varepsilon}\right\| \leqq 2 \sup _{t \leqq T}\left\|u_{t}\right\|, \quad\left\|\omega_{t_{1}}^{\varepsilon}\right\| \leqq 2 \sup _{t \leqq T}\left\|\omega_{t}\right\|
$$

This allows us to obtain the stability and hence the convergence also in the second interval of time. The procedure can be iterated up to the last interval and this concludes the proof.

\section{References}

1. Chorin, A.J.: J. Fluid Mech. 57, 785 (1973)

2. Marchioro, C., Pulvirenti, M.: Vortex methods in two-dimensional fluid mechanics. In: Lecture Notes in Physics, Vol. 203. Berlin, Heidelberg, New York: Springer 1984

3. Chorin, A.J., Hughes, T.J.R., McCracken, M.F., Marsden, J.E.: Commun. Pure Appl. Math. 31, 205 (1978)

4. Marsden, J.: J. Funct. Anal. 13, 51 (1973)

5. Marsden, J.: Bull. Am. Math. Soc. 80, 154 (1974)

6. Benfatto, G., Pulvirenti, M.: Generation of vorticity near the boundary in planar NavierStokes flows. Commun. Math. Phys. 96, 59 (1984)

7. Alessandrini, G., Douglis, A., Fabes, E.: Ann. Mat. Pura Appl. CXXXV, 329 (1983)

8. Ladyzhenskaya, O.A.: The mathematical theory of viscous incompressible flows. New York: Gordon and Breach 1969

9. Temam, R.: Navier-Stokes equations. Amsterdam: North-Holland 1977

Communicated by J. L. Lebowitz

Received November 12, 1985; in revised form March 3, 1986 